\title{
Quadratic autocatalysis with non-linear decay. II: the effect of incomplete mixing
}

\author{
Ahmed Hussein Msmali ${ }^{1} \cdot$ Mark I. Nelson $^{2} \cdot$ Maureen P. Edwards ${ }^{2}$
}

(C) Springer Nature Switzerland AG 2019

\begin{abstract}
Isothermal kinetic models based upon quadratic and cubic autocatalysis have been widely investigated as model schemes for a variety of chemical systems. A standard assumption in such models is that the process is being investigated in a continuously fed well-stirred tank reactor. This allows the mathematical model to be idealised as a system of ordinary differential equations. In this paper we relax the assumption that the reactor is well-mixed and employ an established two-parameter model for incomplete mixing. We use this to investigate the consequences of poor mixing upon the static and dynamic multiplicity of the model. We show that the phenomenon of incomplete mixing can reduce the complexity of the steady-state diagram by removing Hopf and limit-point bifurcations from the system. Incomplete mixing may also increase the complexity of the steady-state diagram through the creation of additional Hopf bifurcation points.
\end{abstract}

Keywords Autocatalysis $\cdot$ Flow reactor $\cdot$ Hopf bifurcation $\cdot$ Incomplete mixing $\cdot$ Singularity theory

\section{Introduction}

Kinetic models based upon quadratic and cubic autocatalysis have been widely investigated as prototype non-linear chemical kinetic schemes since they reveal key aspects of a range of real chemical systems. A standard assumption is that the reactions occur in a well-stirred reactor. The consequence of this is that the mathematical model consists of a system of non-linear differential equations, rather than a system of non-linear partial differential equations. The assumption that the reactions are subject to perfect, or complete, mixing is well motivated, since it is straightforward to obtain these conditions for small-scale experiments carried out in a laboratory.

The assumption of perfect mixing becomes increasingly questionable as the process is scaled up. Although industrial reactions are often carried out in vessels subject to stirring, their contents are typically not well-mixed.
A variety of methods have been developed by industrial chemists and chemical engineers to model the effects of incomplete mixing upon chemical reactions whilst still retaining the paradigm that the system is represented by a system of differential equations ([6, chapter 14$]$, [19, chapter 16]).

In an earlier paper we provided a comprehensive analysis of the static and dynamic multiplicity of a prototype non-linear chemical mechanism: quadratic multiplicity subject to non-linear decay [18] (The meaning of the terms static and dynamic multiplicity is provided in Sect. 2.3). The reaction mechanism for this kinetic scheme is given by

$$
\begin{aligned}
& A+B \rightarrow 2 B, \quad \text { rate }=k_{1} a b, \\
& B \rightarrow C, \quad \text { rate }=\frac{k_{2} b}{1+r b} .
\end{aligned}
$$

$\triangle$ Mark I. Nelson, mnelson@uow.edu.au; Ahmed Hussein Msmali, amsmali@jazanu.edu.sa; Maureen P. Edwards, maureen@uow.edu.au | ${ }^{1}$ Department of Mathematics, College of Science, Jazan University, Jazan, Saudi Arabia. ${ }^{2}$ School of Mathematics and Applied Statistics, University of Wollongong, Wollongong, NSW 2522, Australia. 
This scheme was first investigated, as model for isothermal chain-branch reactions, by Merkin and his co-workers [2, $16,17]$.

We extend our earlier analysis by investigating the effect that incomplete mixing has upon static and dynamic multiplicity. We do this by employing a two-parameter mixing model in which the reactor is split into two compartments. These compartments represent the flowing stream, i.e. a region that is highly agitated and into which the feed enters, and a region that is relatively stagnant, which exchanges material slowly with the flowing stream. Each compartment is modelled as a continuously stirred tank reactor. This widely used approach was introduced by Corrigan and Beavers [3]. It was independently introduced by Kumpinsky \& Epstein to investigate the effects of incomplete mixing transitions in bistable systems [14] and latter used by Ganapathisubramanian to investigate the iodate-As(III) system [7, 8].

Variations on the method are possible. For example, Györgi \& Field used a two compartment model to investigate the effect of imperfect mixing upon the BelousovZhabotinskii reaction [11]. However, they assumed that spatial inhomogeneities were due to the formation of a small region of poor mixing near the tip of one of the feed streams. Thus one of the feed streams enters directly into the 'stagnant region'. Another extension of the model is to increase the number of zones. The network-of-zones model, which includes $2 N^{2}$ zones, can be used to investigate mixing across an entire reactor $[12,13]$. For an introduction into experimental investigations into the effects of stirring and mixing we refer the reader to [4]. It has thus been established for over twenty years that the impact of imperfect mixing is not just an engineering problem associated with large reactors but a phenomenon that can impact experiments at the lab scale. Despite this, some academic physical chemists still consider the influence of imperfect mixing on the behaviour of non-linear chemical systems to be merely "a technology problem"!

The two new parameters associated with the mixing model are the fractional volume associated with the agitated region, $\varepsilon, 0 \leq \varepsilon \leq 1$, and the degree of mixing between the two regions, $\delta$. As the mixing model introduces two additional unfolding parameters we do not analyse fully the model; rather we investigate circumstances under which the phenomena of incomplete mixing can change the static and dynamic multiplicity of the model. Our approach is to first choose parameter values which generate a particular steady-state diagram in the well mixed model. We then investigate, for a fixed size of the stagnant region $(\varepsilon)$, the behaviour of the model as the mixing parameter $(\delta)$ is varied.

\section{Equations for the imperfect mixing model}

\subsection{Dimensional imperfect mixing model}

In this section we provide the model equations for a chemical process governed by quadratic autocatalytic kinetics with catalyst decay subject to imperfect mixing.

Differential equations in the highly agitated region

$V(1-\varepsilon) \frac{\mathrm{d} a_{1}}{\mathrm{~d} t}=q\left(a_{0}-a_{1}\right)-V(1-\varepsilon) k_{1} a_{1} b_{1}-q \delta\left(a_{1}-a_{2}\right)$

$$
\begin{aligned}
V(1-\varepsilon) \frac{\mathrm{d} b_{1}}{\mathrm{~d} t}= & q\left(b_{0}-b_{1}\right)+V(1-\varepsilon) k_{1} a_{1} b_{1} \\
& -V(1-\varepsilon) \frac{k_{2} b_{1}}{1+r b_{1}}-q \delta\left(b_{1}-b_{2}\right) .
\end{aligned}
$$

Differential equations in the stagnant region

$V \varepsilon \frac{\mathrm{d} a_{2}}{\mathrm{~d} t}=q \delta\left(a_{1}-a_{2}\right)-V \varepsilon k_{1} a_{2} b_{2}$

$V \varepsilon \frac{\mathrm{d} b_{2}}{\mathrm{~d} t}=q \delta\left(b_{1}-b_{2}\right)+V \varepsilon k_{1} a_{2} b_{2}-V \varepsilon \frac{k_{2} b_{2}}{1+r b_{2}}$

The parameters and variables in Eqs. (3)-(6) are: $a_{0}$, the reactant concentration in the feed $\left(\mathrm{mol} \mathrm{m}^{-3}\right) ; a_{1}$, the reactant concentration in the agitated region $\left(\mathrm{mol} \mathrm{m}^{-3}\right) ; a_{2}$, the reactant concentration in the stagnant region $\left(\mathrm{mol} \mathrm{m}^{-3}\right)$; $b_{0}$, the autocatalyst concentration in the feed $\left(\mathrm{mol} \mathrm{m}^{-3}\right)$; $b_{1}$, the autocatalyst concentration in the agitated region $\left(\mathrm{mol} \mathrm{m}{ }^{-3}\right) ; b_{2}$, the autocatalyst concentration in the stagnant region ( $\left.\mathrm{mol} \mathrm{m}^{-3}\right) ; k_{1}$, the rate constant for the autocatalytic step, reaction (1), $\left(\mathrm{m}^{3} \mathrm{~mol}^{-1} \mathrm{~s}^{-1}\right) ; k_{2}$, the decay rate, reaction $(2),\left(\mathrm{s}^{-1}\right) ; q$, the flow rate through the reactor $\left(\mathrm{m}^{3} \mathrm{~s}^{-1}\right) ; r$, the surface saturation term, reaction (2), $\left(\mathrm{m}^{3} \mathrm{~mol}^{-1}\right) ; t$, the time $(\mathrm{s}) ; V$, the volume of the reactor $\left(\mathrm{m}^{3}\right)$; $\delta$, the mixing parameter between two regions $(-)$; and $\varepsilon$, $0 \leq \varepsilon<1$, the fraction of the total volume occupied by the stagnant region (-).

In what follows we often refer to the value for the parameter $\varepsilon$ as the (dimensionless) size of the stagnant region.

The main experimental control parameter in Eqs. (3)-(6) is the residence time $\tau(\tau=V / q)$. We will take the scaled version of this quantity as the primary bifurcation parameter.

\subsection{Dimensionless imperfect mixing model}

In this section we scale the imperfect mixing model, Eqs. (3)-(6). To do this we introduce the following 
dimensionless groups: $\alpha_{1}=a_{1} / a_{0}, \alpha_{2}=a_{2} / a_{0}, \beta_{1}=b_{1} / a_{0}$, $\beta_{2}=b_{2} / a_{0}$, and $t^{*}=k_{1} a_{0} t$.

The scaled model is given by

$\frac{\mathrm{d} \alpha_{1}}{\mathrm{~d} t^{*}}=\frac{1-\alpha_{1}}{(1-\varepsilon) \tau}-\alpha_{1} \beta_{1}-\frac{\delta\left(\alpha_{1}-\alpha_{2}\right)}{(1-\varepsilon) \tau}$,

$\frac{\mathrm{d} \beta_{1}}{\mathrm{~d} t^{*}}=\frac{\beta_{0}-\beta_{1}}{(1-\varepsilon) \tau}+\alpha_{1} \beta_{1}-\frac{\kappa_{2} \beta_{1}}{1+\rho \beta_{1}}-\frac{\delta\left(\beta_{1}-\beta_{2}\right)}{(1-\varepsilon) \tau}$,

$\frac{\mathrm{d} \alpha_{2}}{\mathrm{~d} t^{*}}=\frac{\delta\left(\alpha_{1}-\alpha_{2}\right)}{\varepsilon \tau}-\alpha_{2} \beta_{2}$

$\frac{\mathrm{d} \beta_{2}}{\mathrm{~d} t^{*}}=\frac{\delta\left(\beta_{1}-\beta_{2}\right)}{\varepsilon \tau}+\alpha_{2} \beta_{2}-\frac{\kappa_{2} \beta_{2}}{1+\rho \beta_{2}}$,

where the parameter groups are: the non-dimensional concentration of autocatalyst in the feed, $\beta_{0}=b_{0} / a_{0}$; the non-dimensional decay-rate, $\kappa_{2}=k_{2} /\left(k_{1} a_{0}\right)$; the nondimensional saturation term, $\rho=r a_{0}$; and, the non-dimensional residence time $\tau=v k_{1} a_{0} / q$.

In the limit that the mixing parameter approaches zero, $\delta \rightarrow 0$, the stagnant region uncouples from the highly agitated region. This gives a one-parameter incomplete mixing model that is known as the dead space model. The static and dynamic multiplicity of this model is identical to that of the well mixed model. However, the residence time in such a reactor is not the residence time of the well mixed reactor, $\tau^{*}$, but rather it is also related to the size of the dead volume, $(1-\varepsilon) \tau^{*}$. Thus a bifurcation phenomenon that occurs at residence time $\tau^{*}=\tau_{b}^{*}$ in the well mixed model will occur at a higher residence time in the dead volume reactor, $\tau^{*}=\tau_{b}^{*} /(1-\varepsilon)$.

In the limit that the mixing parameter becomes infinitely large, $\delta \rightarrow \infty$, the concentrations of the reactants in the two zones of the reactor become identical. In this limit the static and dynamic multiplicity of the reactor are identical to those of the well stirred reactor.

\subsection{Mathematical techniques}

The main mathematical techniques used to analyse Eqs. (7)-(10) are bifurcation theory and singularity theory. A good introduction to the former for a non-expert is provided by Strogatz [20]. Two excellent texts showing how to apply both sets of techniques are those by Gray and Scott [10] and Ajbar and Alhumaizi [1]. The former emphasises isothermal and non-isothermal problems in non-linear chemical kinetics. The latter emphasises isothermal applications based around the chemostat. The review paper by Gray and Roberts [9] provides an invaluable 'recipe book' for analysing systems of two coupled ordinary differential equations.
Bifurcation theory and singularity theory are tools that can be used to efficiently investigate the static and dynamic multiplicity exhibited by a non-linear model. The most basic kind of solution to such a model is 'steady-state' behaviour, i.e. a solution that is independent of time. Static multiplicity refers to questions such as: 'how many steadystate solutions does a model have?', 'how do the number of solutions vary as one parameter is changed?' and 'if the steady-state solutions are plotted as a function of one parameter what kind of shape do we see?'.

The next simplest kind of solution is a special type of time dependent solution; a periodic solution. The existence of such solutions is closely connected to the phenomena of Hopf bifurcations. Dynamic multiplicity, as used in this paper, asks questions such as:'how many Hopf bifurcations does a model have?', and 'how do the number of Hopf bifurcations vary as one parameter is changed'?

The answers obtained by investigating issues relating to static and dynamics multiplicity can be combined to produce a bifurcation diagram, this is a'map' which can be used to identify where different types of behaviours occur.

The steady-state diagrams were determined using the software package XPPAUT [5]. Standard representations are used, so that solid and dashed lines indicate stable and unstable steady-state solutions. A filled-in box represents a Hopf bifurcation. Where periodic solution branches are shown a filled in circle indicates a stable periodic solution. The location of the circle indicates the 'size' of the periodic solution. This is measured by its L2-norm [5].

\section{Results}

The well-stirred reactor model contains four parameters: $\tau^{*}, \kappa_{2}, \beta_{0}$ and $\rho$. The residence time $\left(\tau^{*}\right)$ is the most easily controlled parameter experimentally, it is therefore taken to be the primarily bifurcation parameter. By convention the value for the scaled saturation term $(\rho)$ is maintained at a constant value $[2,16,17]$. This leaves two parameters which are treated as secondary bifurcation parameters: the scaled decay rate $\left(\kappa_{2}\right)$ and the scaled concentration of the catalyst in the feed $\left(\beta_{0}\right)$. For a full discussion of the static and dynamic multiplicity of this model we refer to [18].

The mixing model introduces a further two parameters: the dimensionless size of the stagnant region $(\varepsilon)$ and the mixing parameter $(\delta)$.

Our strategy in the following is to select a steady-state diagram for the perfect mixing model by choosing appropriate values of the secondary bifurcation parameters $\left(\kappa_{2}, \beta_{0}\right)$. We consider three values for the size of the stagnant region: $\varepsilon=0.1, \varepsilon=0.2$, and $\varepsilon=0.3$. We then investigate how the static and dynamic behaviour of the mixing 
model depends upon the value of the mixing parameter $(\delta)$.

In the limit that $\delta \rightarrow \infty$ we obtain the perfect mixing model. In the limit that $\delta \rightarrow 0$ we obtain the dead volume model which has identical bifurcations to those in the perfect mixing model, but at higher values of the residence time. (See Sect. 2.2.) However, note that the bifurcations of the imperfect mixing model do not necessarily smoothly embed into the dead volume model due to the discontinuity at $\varepsilon=0$.

In Sect. 3.1 we reduce the steady-state problem from four to two non-linear equations. In Sect. 3.2 we outline a methodology that can be applied to determine location of the cusp and isola singularities in the secondary bifurcation parameter plane. In Sect. 3.3 we investigate how the steady-state diagram of the incomplete mixing model can differ from that of the perfect mixing model.

\subsection{Steady-state solutions branches}

In this section we reduce the steady-state analysis of the scaled model, Eqs. (7)-(10), to the study of a system of two coupled algebraic equations.

From Eqs. (7) and (9) we find that

$\alpha_{2}=\frac{\alpha_{1}\left[1+\delta+\beta_{1} \tau(1-\varepsilon)\right]-1}{\delta}$,

$\beta_{2}=-\frac{\delta\left[1-\alpha_{1}-(1-\varepsilon) \alpha_{1} \tau \beta_{1}\right]}{\tau \varepsilon\left[1-\alpha_{1}(1+\delta)-(1-\varepsilon) \alpha_{1} \tau \beta_{1}\right]}$.

Using (11) and (12) the steady-state problem reduces to a system of two algebraic equations given by

$A-B=0$,

$\frac{B}{\delta}-C-E=0$,

where

$A=-\frac{\left[1-\alpha_{1} \tau(1-\varepsilon)\right] \rho \beta_{1}^{2}+\left[1-\beta_{0} \rho+\tau(1-\varepsilon)\left(\kappa_{2}-\alpha_{1}\right)\right] \beta_{1}-\beta_{0}}{\tau(1-\varepsilon)\left(1+\rho \beta_{1}\right)}$,

$B=\frac{\delta\left[(1-\varepsilon) \tau^{2} \varepsilon \alpha_{1} \beta_{1}^{2}+\left(\delta \alpha_{1}-\varepsilon+\varepsilon \alpha_{1}\right) \tau \beta_{1}-\delta\left(1-\alpha_{1}\right)\right]}{\tau^{2} \varepsilon\left[(1-\varepsilon) \alpha_{1} \tau \beta_{1}-1+\alpha_{1}(1+\delta)\right](1-\varepsilon)}$,

$C=\frac{\left[\alpha_{1}\left\{1+\delta+\beta_{1} \tau(1-\varepsilon)\right\}-1\right]\left[1-\alpha_{1}-(1-\varepsilon) \alpha_{1} \tau \beta_{1}\right]}{\tau \varepsilon\left[1-\alpha_{1}(1+\delta)-(1-\varepsilon) \alpha_{1} \tau \beta_{1}\right]}$,

$E=\frac{1-\alpha_{1}-(1-\varepsilon) \alpha_{1} \tau \beta_{1}}{\tau \alpha_{1}(1-\varepsilon)(\tau \varepsilon-\delta \rho) \beta_{1}+(\delta \rho-\tau \varepsilon)\left(1-\alpha_{1}\right)+\tau \varepsilon \delta \alpha_{1}}$.

It is not possible to reduce the system (7)-(10) any further. The steady-state solution of Eqs. (13) and (14) can be investigated using the method outlined in [9]. It is important to appreciate that the analysis of the static multiplicity outlined in [9] applies to any system of two equations regardless of the number of differential equations initially (in our model four).

A special case of interest is when there is no catalyst in the feed, i.e. when $\beta_{0}=0$. In this case the system of Eqs. (13)-(14) reduces to

$\widehat{A}-B=0$,

$\frac{B}{\delta}-C-E=0$,

where

$\widehat{A}=-\frac{\left[1-\alpha_{1} \tau(1-\varepsilon)\right] \rho \beta_{1}^{2}+\left[1+\tau(1-\varepsilon)\left(\kappa_{2}-\alpha_{1}\right)\right] \beta_{1}}{\tau(1-\varepsilon)\left(1+\rho \beta_{1}\right)}$.

In this case the steady-state solutions includes a washout solution

$\left(\alpha_{1}, \beta_{1}, \alpha_{2}, \beta_{2}\right)=(1,0,1,0)$.

\subsection{Static multiplicity}

In the previous section we reduced the steady-state problem from a system of four Eqs. (7)-(10) to a system of two Eqs. (13) and (14). The latter can be written in the general form

$f\left(\alpha_{1}, \beta_{1}, \tau, \kappa_{2}, \beta_{0}, \varepsilon, \delta, \rho\right)=0$,

$g\left(\alpha_{1}, \beta_{1}, \tau, \kappa_{2}, \beta_{0}, \varepsilon, \delta, \rho\right)=0$.

This system contains two state variables $\left(\alpha_{1}\right.$ and $\left.\beta_{1}\right)$, a primary bifurcation parameter $(\tau)$, four secondary bifurcation parameters, $\left(\kappa_{2}, \beta_{0}, \varepsilon\right.$, and $\left.\delta\right)$ and one tertiary bifurcation parameter $(\rho)$. Suppose that at the point

$(\alpha, \beta, \tau)=\left(\alpha_{1}, \beta_{1}, \tau\right)$

there is either a cusp or isola singularity. Then the following conditions hold [9].

Cusp singularity:

$f=g=\frac{\mathrm{d} \tau}{\mathrm{d} \alpha_{1}}=\frac{\mathrm{d}^{2} \tau}{\mathrm{d} \alpha_{1}{ }^{2}}=0$.

Non-degeneracy conditions

$\frac{\mathrm{d}^{3} \tau}{\mathrm{d} \alpha_{1}{ }^{3}} \neq 0$ and $\quad \frac{\mathrm{d} \alpha_{1}}{\mathrm{~d} \tau} \neq 0$.

Isola singularity:

$f=g=\frac{\mathrm{d} \tau}{\mathrm{d} \alpha_{1}}=\frac{\mathrm{d} \alpha_{1}}{\mathrm{~d} \tau}=0$. 
Non-degeneracy conditions

$\frac{\mathrm{d}^{2} \tau}{\mathrm{d} \alpha_{1}^{2}} \neq 0 \quad$ and $\quad \frac{\mathrm{d}^{2} \tau}{\mathrm{d} \alpha_{1}^{2}} \neq 0$

Figure 1 shows the cusp curves for both the ideal mixing model $(\delta=\infty$, black line) and the imperfect mixing model $(\delta=0.2)$, for three sizes of the dimensionless stagnant region $(\varepsilon)$. As the size of the dimensionless stagnant region $(\varepsilon)$ increases the cusp singularity curves are not fully contained within each other. For instance, the curve when $\varepsilon=0.3$ intersects the curve when $\varepsilon=0.2$ at several locations.

Figure 2 shows the isola curves for the perfect mixing model ( $\delta=\infty$, black line) and the imperfect mixing model, with $\delta=0.2$, for three sizes of the dimensionless stagnant regions $(\varepsilon)$. We again see that the curves can intersect each other. Note that the curve when $\varepsilon=0.3$ is 'smaller' than the curve when $\varepsilon=0.1$, but 'higher' than that when $\varepsilon=0.2$.

\subsection{Steady-state diagrams}

In the following we fix values for the secondary bifurcation parameters $\left(\kappa_{2}, \beta_{0}\right)$ to select a particular steady-state diagram in the well-mixed case. In what follows there are five base cases, as in the perfect mixing model there are five generic static steady-state diagrams [18]. We then investigate how the phenomenon of incomplete mixing can change the steady-state diagram. In this section our investigation is built around the steady-state diagrams for the well mixed case. We take as default parameter values $\delta=0.2$ and $r=10$ and the consider the cases $\varepsilon=0.1$, $\varepsilon=0.2$, and $\varepsilon=0.3$. The three incomplete mixing models

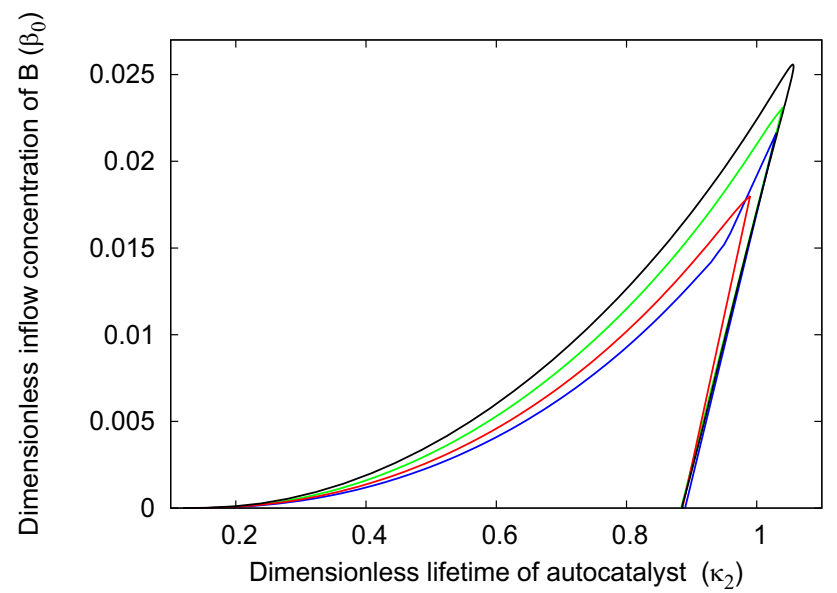

Fig. 1 The cusp singularity in the secondary bifurcation parameter plane. Parameter values: $\rho=10, \delta=0.2, \varepsilon=0.1$ (green line), $\varepsilon=0.2$ (blue line), $\varepsilon=0.3$ (red line) and $\varepsilon=0$ and $\delta=\infty$ (black line). Part of this figure is blown up in Figs. 8 and 13

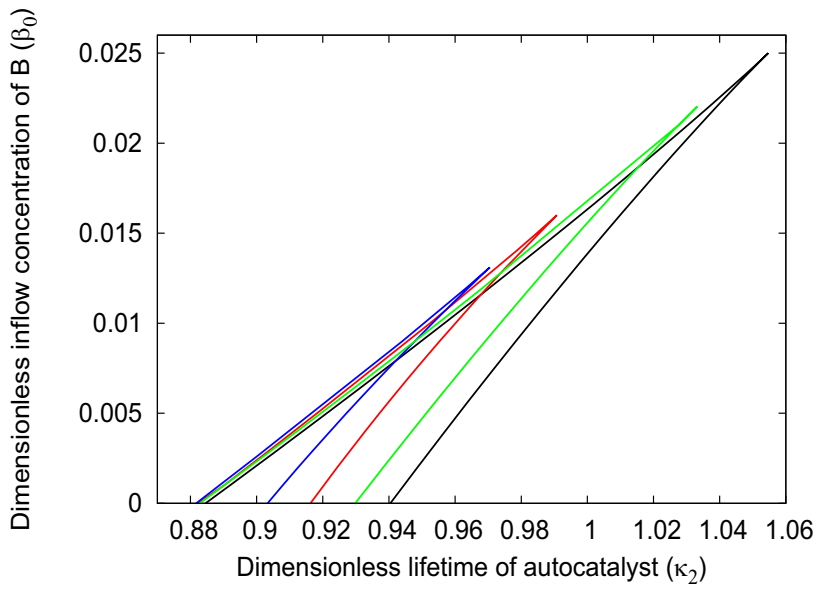

Fig. 2 The isola singularity in the secondary bifurcation parameter plane. Parameter values: as in Fig. 1

are represented by green, blue, and red curves respectively in all figures. The perfect mixing model is represented by a black curve.

\subsubsection{Unique steady-state diagram}

In this section we set the values of the secondary bifurcation parameters, the scaled decay parameter $\kappa_{2}$ and the scaled catalyst concentration in the feed $\beta_{0}$, so that the steady-state diagram in the case of perfect mixing is the unique steady-state diagram, called type 'U' in [18]. In Fig. 3 this steady-state diagram is indicated by the the black line. Its distinguishing feature is that for any value of the primary bifurcation parameter there is a unique steady-state

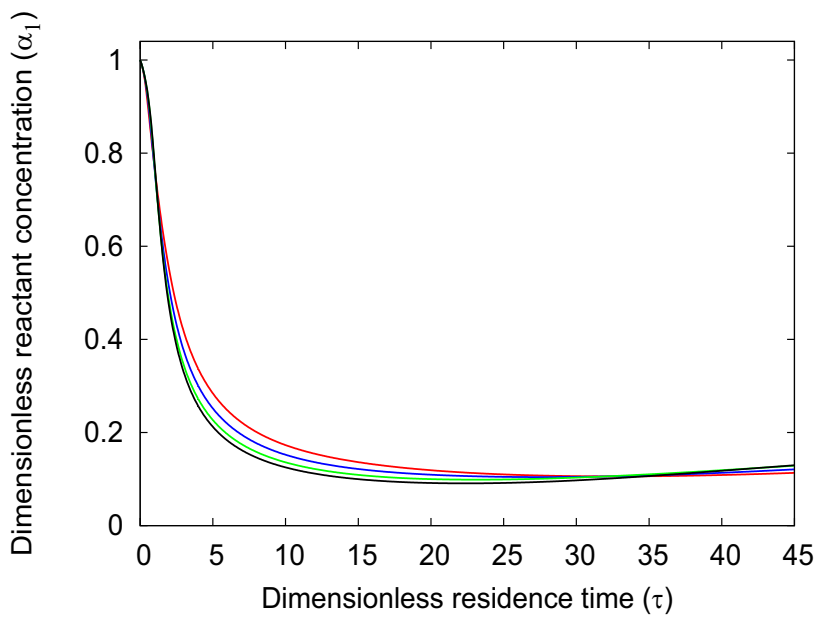

Fig. 3 Steady-state diagrams. In the well mixed case (black line) the steady-state diagram type is unique. The green $(\varepsilon=0.1)$, blue $(\varepsilon=0.2)$, and red $(\varepsilon=0.3)$ curves represent cases of incomplete mixing. Secondary bifurcation parameter values: $\left(\kappa_{2}, \beta_{0}\right)=(0.3,0.087)$. Other parameter values as in Fig. 1 


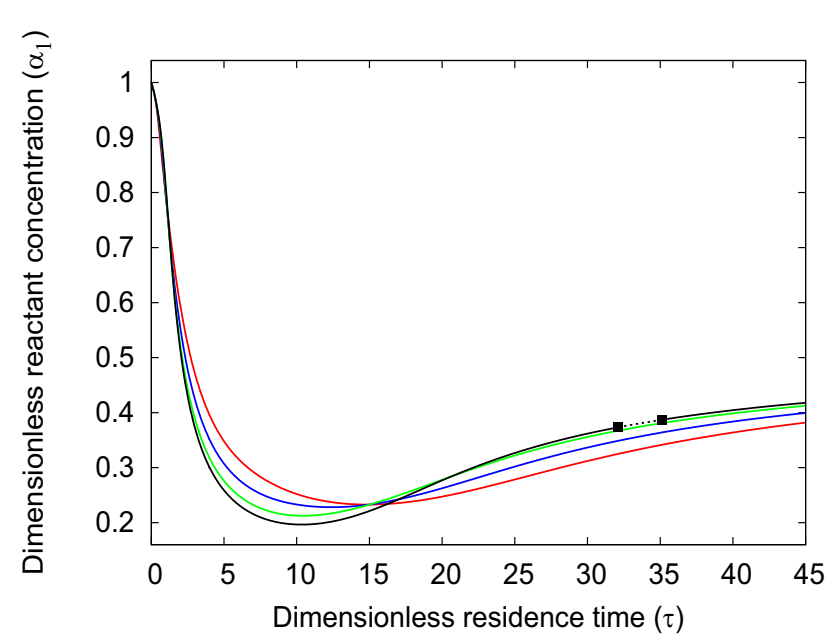

Fig. 4 Steady-state diagrams. In the well mixed case (black line) the steady-state diagram type is unique with two Hopf bifurcation points. The green $(\varepsilon=0.1)$, blue $(\varepsilon=0.2)$, and red $(\varepsilon=0.3)$ curves represent cases of incomplete mixing. Secondary bifurcation parameter values: $\left(\kappa_{2}, \beta_{0}\right)=(0.6,0.087)$. Other parameter values as in Fig. 1

solution. For the chosen values of the two mixing parameters $(\varepsilon, \delta)$ there is no substantive change in the behaviour of the reactor.

In Fig. 4 the values of the secondary bifurcation parameters have been changed so that the steady-state diagram in the perfect mixing model (the black line) contains two Hopf bifurcation points, called the ' $U+2 \mathrm{H}$ ' steady-state in [18]. Note that the periodic solution branches are not shown. The significance of this figure is that the Hopf bifurcation points do not exist on the incomplete mixing steady-state diagrams, i.e. the phenomenon of incomplete mixing has eliminated periodicity from the system.

In Fig. 5 the value of the mixing parameter is increased from the default value $\delta=0.2$, as used in Fig. 4, to $\delta=15$. Now the steady-state diagram for all three of the incomplete mixing cases retain their Hopf bifurcation points. To more clearly show the location of the Hopf bifurcation Fig. $5 \mathrm{~b}$ blows up the required region from Fig. 4.

We know from Fig. 4 that when the mixing parameter takes value $\delta=0.2$ that none of the three incomplete mixing steady-state diagrams exhibits Hopf bifurcation points. We see from Fig. $5 b$ that when the value of the mixing parameter is increased to $\delta=15$ that all three of the incomplete mixing steady-state diagrams cases exhibit Hopf bifurcation points. We deduce that as the value of the mixing parameter is decreased from $\delta=15$ that there must be critical values at which the Hopf bifurcation points are removed from the steady-state diagram.

In Fig. 6 we unfold the Hopf bifurcation points from the incomplete mixing steady-state diagrams as a function

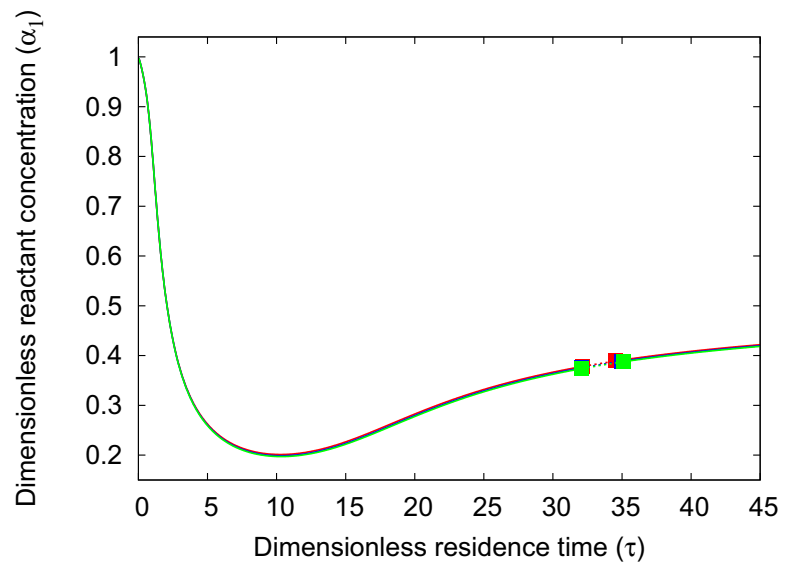

(a) $\mathrm{U}+2 \mathrm{H}$

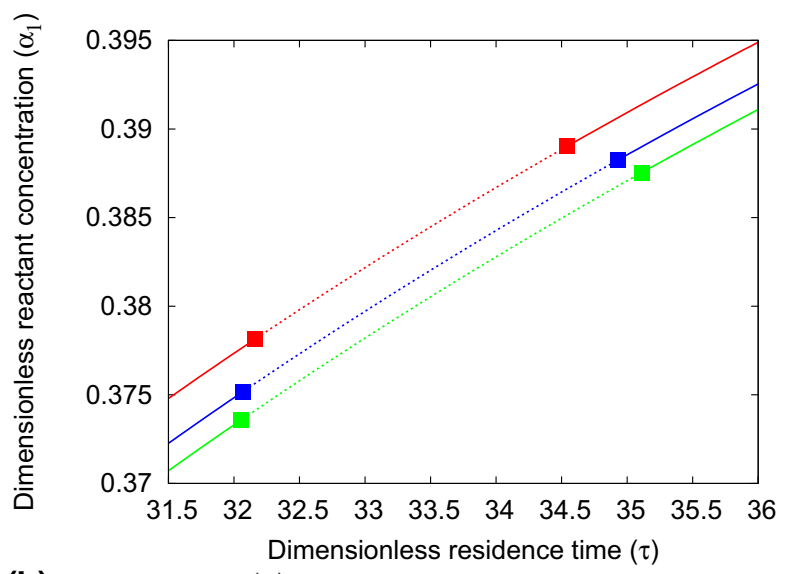

(b) Blow-up of (a) showing the two Hopf bifurcation points

Fig. 5 Steady-state diagrams. In the well mixed case (black line) the steady-state diagram type is unique with two Hopf bifurcation points. The green $(\varepsilon=0.1)$, blue $(\varepsilon=0.2)$, and red $(\varepsilon=0.3)$ curves represent cases of incomplete mixing. Parameter values: $\left(\kappa_{2}, \beta_{0}\right)=(0.6,0.087), \delta=15$ (incomplete mixing curves). Other parameter values as in Fig. 1

of the mixing parameter $(\delta)$. For each of the three cases there is a critical value of the mixing parameter, $\delta_{\mathrm{cr}}$ corresponding to a double-Hopf bifurcation. These critical values are $\delta_{\mathrm{cr}}=2.02(\varepsilon=0.1), \delta_{\mathrm{cr}}=5.44(\varepsilon=0.2)$ and $\delta_{\mathrm{cr}}=9.34(\varepsilon=0.3)$. Thus the critical value of the mixing parameter $\left(\delta_{\mathrm{cr}}\right)$ increases as the size of the dimensionless stagnant region $\varepsilon$ increases.

To conclude, we have seen in this section that a consequence of incomplete mixing, i.e. a sufficiently low value for the mixing parameter $\delta$, is the removal of periodicity through the destruction of Hopf bifurcation points through a double-Hopf bifurcation. From Fig. 6 we see that as the size of the stagnant region increases the minimum value of the mixing parameter required to exhibit Hopf bifurcations also increases. 


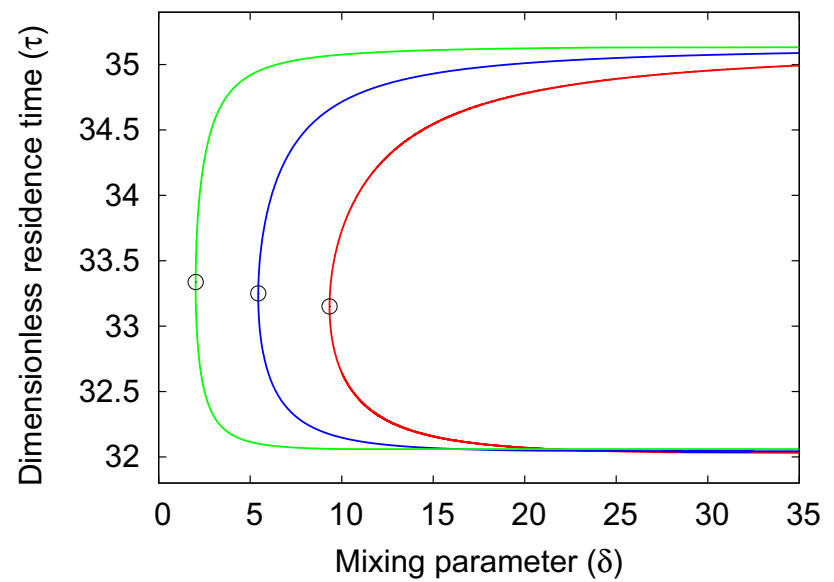

Fig. 6 Unfolding diagram for the Hopf bifurcation points in Fig. 5b. The circles denote the location of double-Hopf bifurcations: $\quad\left(\delta_{\mathrm{cr}}, \tau_{\mathrm{cr}}\right)=(2.02,33.34)$, when $\varepsilon=0.1 \quad$ (green line); $\left(\delta_{\mathrm{cr}}, \tau_{\mathrm{cr}}\right)=(5.44,33.25)$, when $\varepsilon=0.2 \quad$ (blue line); and $\left(\delta_{\mathrm{cr}}, \tau_{\mathrm{cr}}\right)=(9.34,33.15)$ when $\varepsilon=0.3$ (red line)

\subsubsection{Breaking wave steady-state diagram}

In this section we set the values of the secondary bifurcation parameters, the scaled decay parameter $\kappa_{2}$ and the scaled catalyst concentration in the feed $\beta_{0}$, so that the steady-state diagram in the case of perfect mixing is the breaking wave steady-state diagram, called type ' $B$ ' in [18]. In Fig. 7 this steady-state diagram is indicated by the the black line. Its distinguishing feature is that it consists of

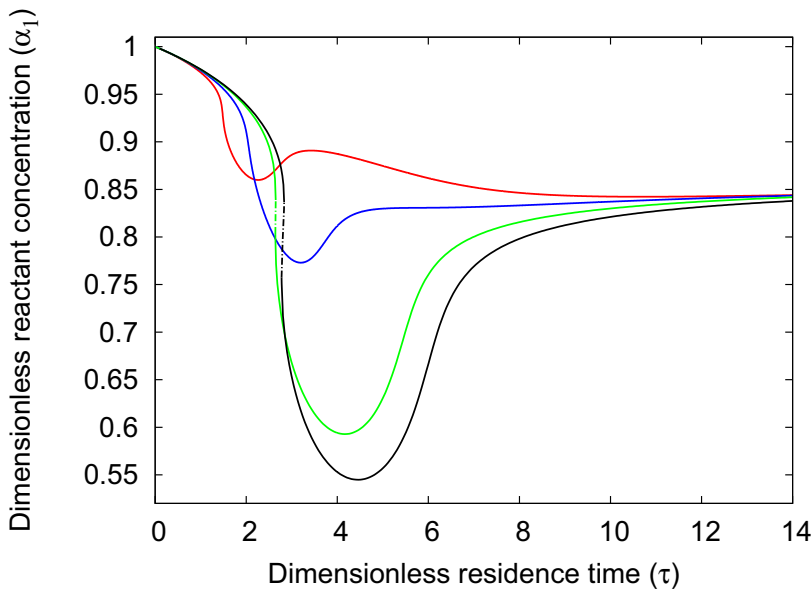

Fig. 7 Steady-state diagrams. In the well-mixed case (black line) the steady-state diagram is a breaking wave. The green $(\varepsilon=0.1)$, blue $(\varepsilon=0.2)$, and red $(\varepsilon=0.3)$ curves represent cases of incomplete mixing. As the size of the dimensionless stagnant region increases from $\varepsilon=0.1$ (green line) to $\varepsilon=0.2$ (blue line) the static steadystate diagram transitions from a breaking wave to an unique steady-state diagram. Secondary bifurcation parameter values: $\left(\kappa_{2}, \beta_{0}\right)=(0.99,0.02)$. Other parameter values as in Fig. 1 a single branch of solutions containing two limit-point bifurcations.

In Fig. 7 the behaviour of the three incomplete mixing systems depends upon the size of the stagnant region $(\varepsilon)$. If the stagnant region is sufficiently small then the steadystate type is a breaking wave ( $\varepsilon=0.1$, green line). However, if the stagnant region is sufficiently large then the steadystate diagram type becomes 'unique' ( $\varepsilon=0.2$, blue line; $\varepsilon=0.3$, red line). This shows that the incomplete mixing may change the static steady-state diagram.

Figure 8 shows a blow-up of the cusp singularity curve, Fig. 1 . The red circle, $\left(\kappa_{2}, \beta_{0}\right)=(0.99,0.02)$, corresponds to the choice of secondary bifurcation parameters used in Fig. 7. It is clear that this point is outside the region of hysteresis for the systems with a larger dimensionless stagnant region ( $\varepsilon=0.2$, blue line; $\varepsilon=0.3$, red line). This explains why in Fig. 7 the static steady-state diagram is a breaking wave only for the smaller sized stagnant region $(\varepsilon=0.1)$.

In Fig. 9 the value of the mixing parameter is increased from $\delta=0.2$ to $\delta=2$. Now the steady-state diagram in all three cases is a breaking wave, i.e. increasing the mixing parameter overcomes the negative effects associated with a larger stagnant region. This is more clearly seen in Fig. 9b, in which the region containing the limit points is blown up.

We know that for the higher value of the mixing parameter $(\delta=2)$ all of the incomplete mixing steady-state diagrams have two limit points, Fig. 9b, but that when mixing is less efficient, $\delta=0.2$, only one of the steady-state diagrams has two limit points. Hence when $\varepsilon=0.2$ or $\varepsilon=0.3$ there must be a critical value of the mixing parameter $(\delta)$, with $0.2<\delta_{\mathrm{cr}}<2$, at which the limit points are removed from the steady-state diagram.

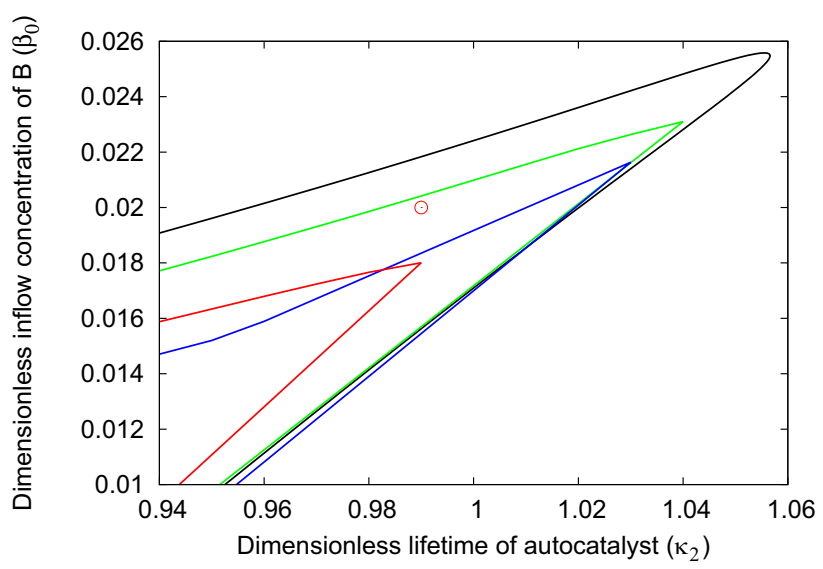

Fig. 8 Blow-up of the cusp singularity curve, Fig. 1. The green $(\varepsilon=0.1)$, blue $(\varepsilon=0.2)$, and red $(\varepsilon=0.3)$ curves represent cases of incomplete mixing. The black curve represents the case of perfect mixing. The red circle, with coordinates $\left(\kappa_{2}, \beta_{0}\right)=(0.99,0.02)$, indicates the parameter values used in Fig. 7 


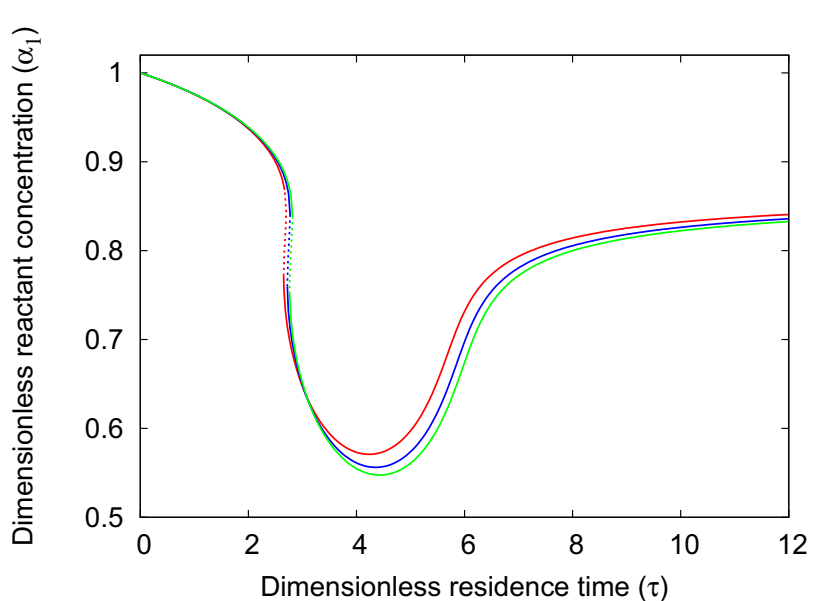

(a)

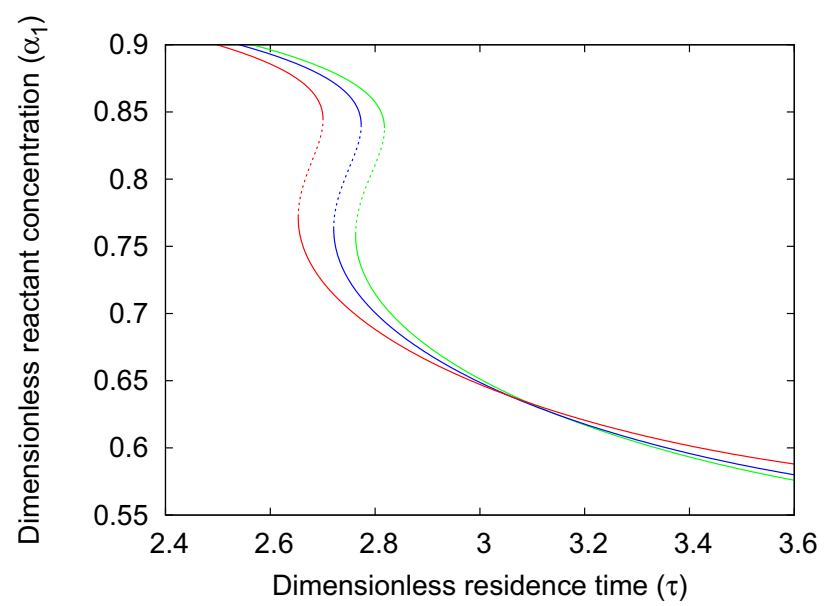

(b) Blow-up figure (a)

Fig. 9 Steady-state diagrams. In the well-mixed case (black line) the steady-state diagram is a breaking wave. The green $(\varepsilon=0.1)$, blue $(\varepsilon=0.2)$, and red $(\varepsilon=0.3)$ curves represent cases of incomplete mixing. Secondary bifurcation parameter values: $\left(\kappa_{2}, \beta_{0}\right)=(0.99,0.02), \delta=2$ (incomplete mixing curves). Other parameter values as in Fig. 1

To investigate this in Fig. 10 we unfold the limit points from Fig. 9b with the mixing parameter $(\delta)$. For each size of the stagnant region $(\varepsilon)$, there is a critical value of the mixing parameter corresponding to a cusp singularity. These critical values are $\delta_{\mathrm{cr}}=0.18(\varepsilon=0.1), \delta_{\mathrm{cr}}=0.38(\varepsilon=0.2)$ and $\delta_{\mathrm{cr}}=0.54(\varepsilon=0.3)$. If the value of the mixing parameter is below its critical value then mixing is not able to overcome the negative effects of the stagnant region and the steady-state diagram is unique. These values explain why in Fig. 7 there are no limit points on the steady-state diagram for the cases $\varepsilon=0.2$ (blue line) and $\varepsilon=0.3$ (red line) and two limit points for the case $\varepsilon=0.1$ (green line). The critical value for the first case, $\varepsilon=0.1$, shows that if the value for the mixing parameter was reduced to below $\delta=0.18$ then the corresponding steady-state diagram

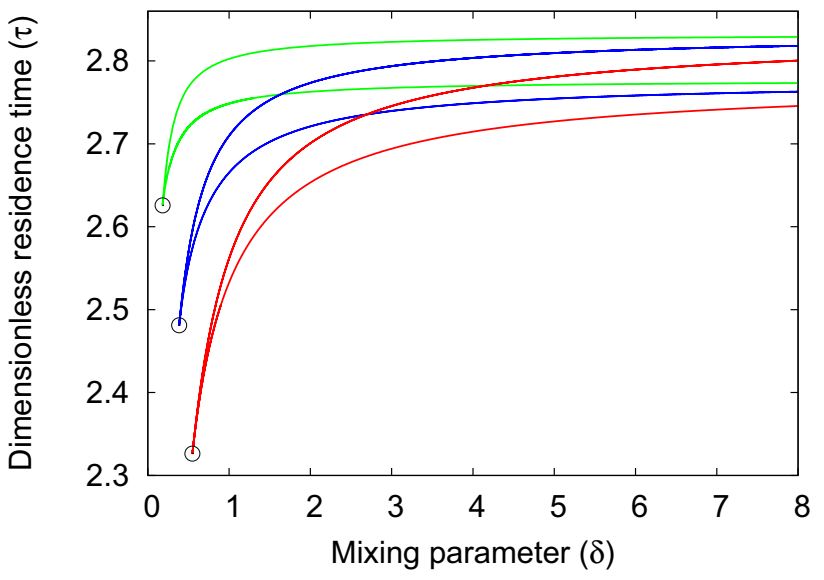

Fig. 10 Unfolding diagram for the limit points in Fig. 9. The circles denote the critical value of the mixing parameters: $\quad\left(\delta_{\mathrm{cr}}, \tau_{\mathrm{cr}}\right)=(0.18,2.62)$, when $\varepsilon=0.1 \quad$ (green line); $\left(\delta_{\mathrm{cr}}, \tau_{\mathrm{cr}}\right)=(0.38,2.48)$, when $\varepsilon=0.2 \quad$ (blue line), and; $\left(\delta_{\mathrm{cr}}, \tau_{\mathrm{cr}}\right)=(0.54,2.32)$, when $\varepsilon=0.3$ (red line)

would contain no limit points. Finally, we note that the critical value of the mixing parameter $\left(\delta_{\mathrm{cr}}\right)$ increases as the value of the stagnant region $\varepsilon$ increases.

In Fig. 11 the values of the secondary bifurcation parameters have been changed so that the steady-state diagram in the perfect mixing model (the black line) contains two Hopf bifurcation points, called the ' $\mathrm{B}+2 \mathrm{H}^{\prime}$ steady-state in [18]. On each of the imperfect mixing steady-state diagrams there are also two Hopf bifurcation points (Note that the periodic solution branches are not shown). The steady-state diagram is a breaking wave when $\varepsilon=0.1$ and $\varepsilon=0.3$. However, it is a unique steady-state diagram when $\varepsilon=0.2$. In Fig. $11 \mathrm{~b}$ the steady-state diagram is blown-up to more clearly show the limit points. For the case $\varepsilon=0.3$ the limit points occur in the very small region indicated by yellow shading.

It appears paradoxically that the first reduction in the size of the stagnant region, from $\varepsilon=0.3$ to $\varepsilon=0.2$, should eliminate the limit points whereas the second reduction, from $\varepsilon=0.2$ to $\varepsilon=0.1$, reintroduces them. However, the discussion of Fig. 1 should be recalled. Viewed as functions of the size of the stagnant region $(\varepsilon)$ the cusp curves are not Russian dolls, the curves for the smaller regions are not contained within the curves for the larger regions. Rather the curves may intersect one-another.

In Fig. 12 the value of the mixing parameter has been increased from $\delta=0.2$, in Fig. 11, to $\delta=2$. Unlike the earlier case the incomplete mixing steady-state diagrams are all now of type ' $\mathrm{B}+2 \mathrm{H}$ '. The location of the limit points is more clearly seen in Fig. $12 b$.

Figure 13 shows a blow-up of the cusp singularity curve, Fig. 1 . The location of the point $\left(\kappa_{2}, \beta_{0}\right)=(0.8,0.01)$, corresponding to the value used in Fig. 12 is shown. The figure 


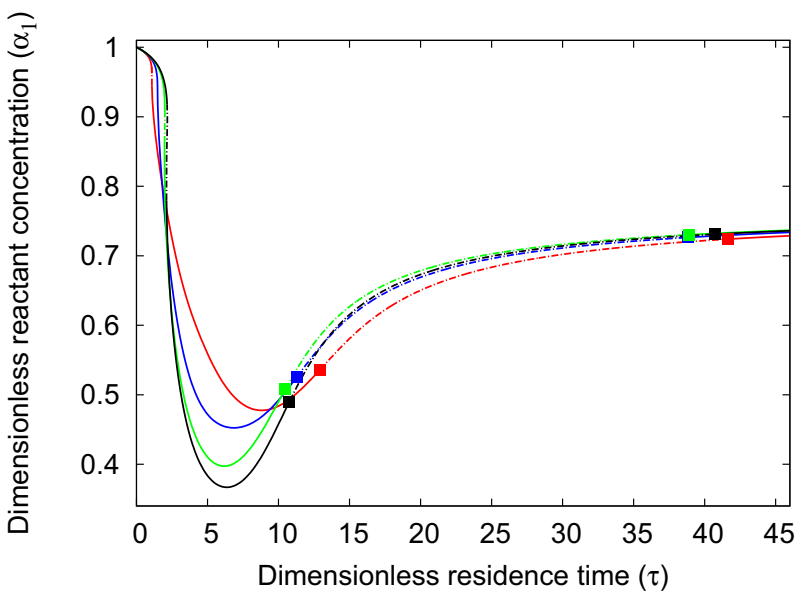

(a)

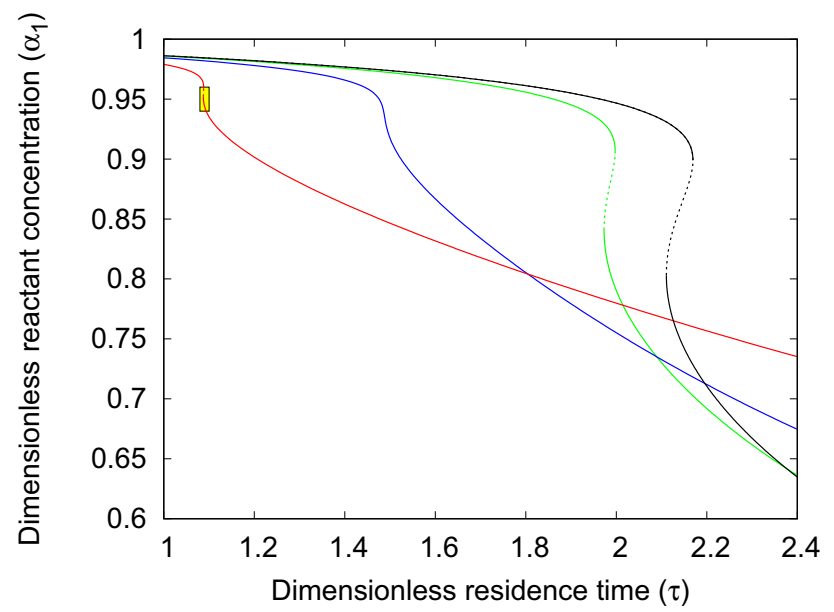

(b) Blow-up of (a)

Fig. 11 Steady-state diagram. In the well mixed case (black line) the steady-state diagram is a breaking-wave with two Hopf bifurcation points. The green $(\varepsilon=0.1)$, blue $(\varepsilon=0.2)$, and red $(\varepsilon=0.3)$ curves represent cases of incomplete mixing. Parameter values: $\left(\kappa_{2}, \beta_{0}\right)=(0.8,0.01)$. Other parameter values as in Fig. 1

shows that this point is outside the hysteresis region when $\varepsilon=0.2$. The figure shows why in Fig. 12 the static steadystate diagram is of the unique type when $\varepsilon=0.2$.

The point is inside the hysteresis regions for the cases $\varepsilon=0.1$ and $\varepsilon=0.3$. For the latter case the point is very near to the edge of the hysteresis region (the red line). Thus the hysteresis region on the steady-state diagram is expected to be smaller than the former case.

We have seen that, fixing the values of the secondary bifurcation parameters to $\kappa_{2}=0.8$ and $\beta_{0}=0.01$, when $\delta=2$ the three incomplete mixing steady-state diagrams have two limit points, Fig. 12. When the mixing parameter is reduced to $\delta=0.2$ the incomplete mixing steady-state diagrams have either zero $(\varepsilon=0.2)$ or two limit points $(\varepsilon=0.1 \& \varepsilon=0.3)$, Fig. 11 . In all cases there are two Hopf bifurcation points.

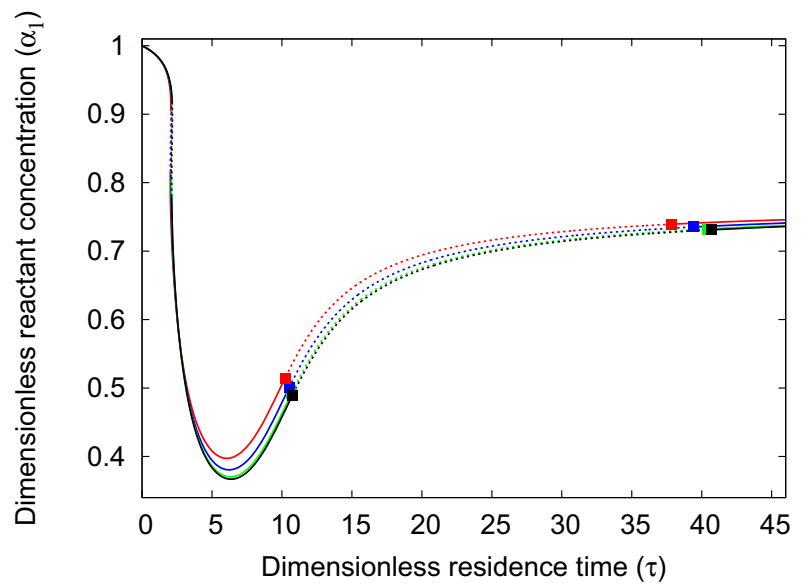

(a)

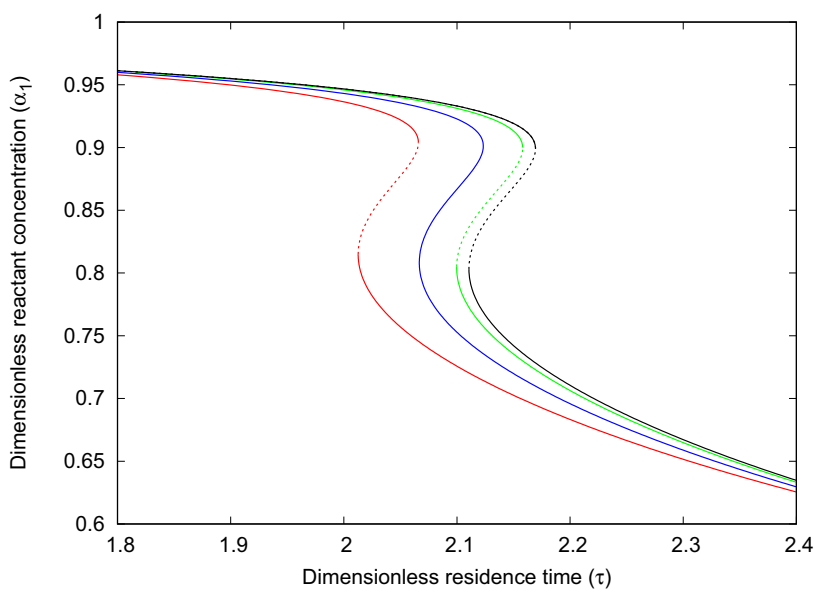

(b) Blow-up of (a)

Fig. 12 Steady-state diagrams. In the well mixed case (black line) the steady-state diagram is a breaking-wave with two Hopf bifurcation points. The green $(\varepsilon=0.1)$, blue $(\varepsilon=0.2)$, and red $(\varepsilon=0.3)$ curves represent cases of incomplete mixing. Parameter values: $\left(\kappa_{2}, \beta_{0}\right)=(0.8,0.01), \delta=2$ (incomplete mixing figures)

We deduce that, for the case when $\varepsilon=0.2$, there must be a critical value for the mixing parameter $(\delta)$ at which the limit points are removed from the steady-state diagram. To investigate this we unfold the limit points for all three scenarios of incomplete mixing shown in Fig. 12. The unfolding diagram is shown in Fig. 14. For each size of the stagnant region $(\varepsilon)$, there is a critical value of the mixing parameter corresponding to a cusp singularity. These critical values are: $\delta_{\mathrm{cr}}=0.15(\varepsilon=0.1) ; \delta_{\mathrm{cr}}=0.27(\varepsilon=0.2)$; and $\delta_{\text {cr }}=0.22 \times 10^{-4}(\varepsilon=0.3)$.

In Fig. 14 the location of the cusp singularity is identified for three sizes of the stagnant region. In Fig. 15 we unfold the cusp singularity in the incomplete mixing plane. The horizontal cut at $\delta=0.2$ reveals that the steady-state diagram has two limit points if either the stagnant region is sufficiently small, $0<\varepsilon<0.136$, or 


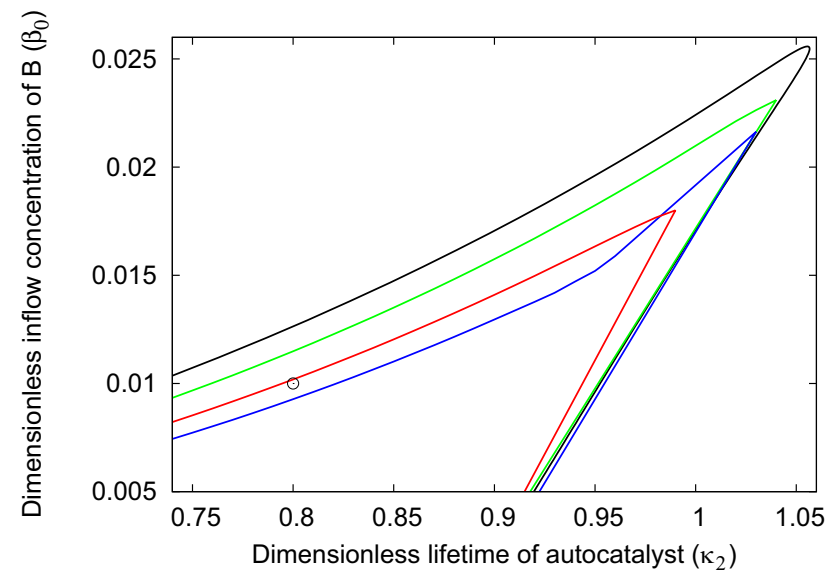

Fig. 13 Blow-up of the cusp singularity curve, Fig. 1. The green $(\varepsilon=0.1)$, blue $(\varepsilon=0.2)$, and red $(\varepsilon=0.3)$ curves represent cases of incomplete mixing. The black curve represents the case of perfect mixing. The black circle, coordinates $\left(\kappa_{2}, \beta_{0}\right)=(0.8,0.01)$, indicates the parameter values used in Fig. 12. Other parameter values as in Fig. 1

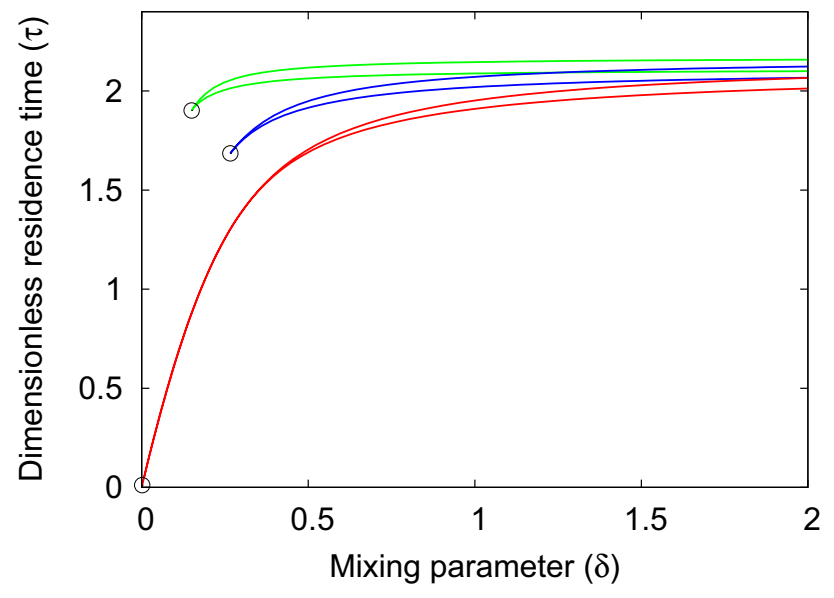

Fig. 14 Unfolding diagram for the limit points in Fig. 12. Parameter values: $\left(\kappa_{2}, \beta_{0}\right)=(0.8,0.01)$. Other parameter values as in Fig. 1. The circles denote the critical value of the mixing parameter at a cusp singularity: $\left(\delta_{\mathrm{cr}}, \tau_{\mathrm{cr}}\right)=(0.15,1.90)$, when $\varepsilon=0.1$ (green curve); $\left(\delta_{\mathrm{cr}}, \tau_{\mathrm{cr}}\right)=(0.27,1.69)$, when $\varepsilon=0.2$ (blue curve); and $\left(\delta_{\mathrm{cr}}, \tau_{\mathrm{cr}}\right)=\left(0.22 \times 10^{-4}, 0.05\right)$, when $\varepsilon=0.3$ (red curve)

sufficiently large, $\varepsilon>0.288$. For intermediate values, $0.137<\varepsilon<0.288$, there are no limit points. This clarifies the behaviour shown in Fig. 9.

Both sets of steady-state diagrams, for $\delta=0.2$ and $\delta=2$, Figs. 11 and 12 contained two Hopf bifurcation points. Figure 16 unfolds these points as a function of the mixing parameter. This shows that poor mixing, i.e. small value of the mixing parameter $(\delta \leq 5)$, does not eliminate periodicity. However, as the mixing parameter becomes sufficiently small the value of the

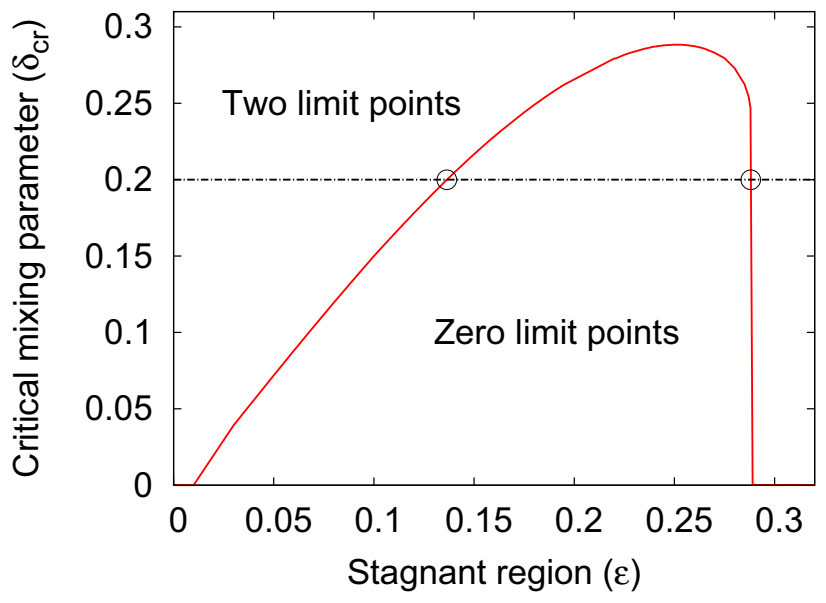

Fig. 15 Unfolding diagram for the cusp singularity point. Parameter values: $\left(\kappa_{2}, \beta_{0}\right)=(0.8,0.01)$. Other parameter values as in Fig. 1. The black circles are $\left(\varepsilon_{\mathrm{cr}}, \delta_{\mathrm{cr}}\right)=(0.136,0.2)$ and $\left(\varepsilon_{\mathrm{cr}}, \delta_{\mathrm{cr}}\right)=(0.288,0.2)$

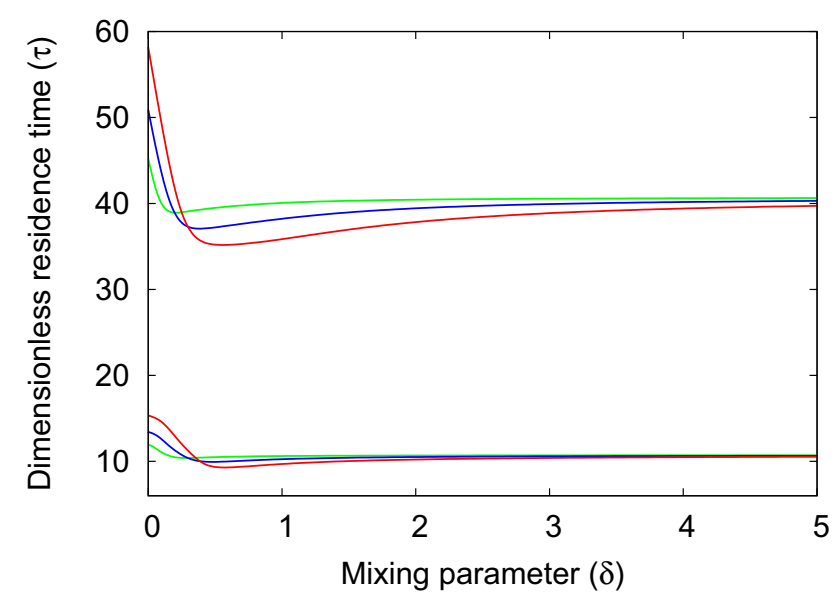

Fig. 16 Unfolding diagram for the Hopf bifurcation points in Figs. 11 and 12. The curves correspond to stagnant fractions: $\varepsilon=0.1$ (green line), $\varepsilon=0.2$ (blue line), and $\varepsilon=0.3$ (red line)

dimensionless residence time at which the Hopf bifurcation occurs begins to increase significantly.

To conclude, in this section we have seen that the effect of incomplete mixing can be to reduce the complexity of the static steady-state diagram from a breaking-wave to the unique type (Fig. 7). Furthermore, as the singularity curves are not contained within one another, the dependence of the steady-state diagram upon the values of the two mixing parameters is not straight forward (Fig. 15).

\subsubsection{Isola steady-state diagram}

In this section we set the values of the secondary bifurcation parameters, the scaled decay parameter $\kappa_{2}$ and the scaled catalyst concentration in the feed $\beta_{0}$, so that the 
steady-state diagram in the case of perfect mixing is the isola steady-state diagram, called type 'I' in [18]. In Fig. 17 this steady-state diagram is indicated by the black line. Its distinguishing feature is that it consists of two disconnected solution branches. One branch consists of the 'unique' steady-state type. The second branch is an isola, i.e. a closed curve. For the chosen parameter values of the mixing model the incomplete mixing steady-state diagrams are also of type I.

The isola steady-state diagram has two limit point bifurcations. Figure 18 shows the unfolding diagram for these bifurcation points for the incomplete mixing steady-state diagrams in Fig. 17. Unlike the two cases considered for the breaking wave, Figs. 10 and 14, in this case reducing the mixing parameter does not change the static steady-state diagram and there are two limit points for any value of the mixing parameter $(0<\delta \leq 2)$.

In Fig. 17 the limit-point bifurcations in the well-mixed case are at $\tau^{*}=3.07$ and $\tau^{*}=6.60$. Therefore the limitpoint bifurcations in the dead reactor volume model, corresponding to the limit $\delta=0$ are at: $3.41 \& 7.34$, when $\varepsilon=0.1 ; 3.84 \& 8.25$, when $\varepsilon=0.2$; and $4.39 \& 9.43$, when $\varepsilon=0.3$. (See the discussion in Sect. 2.20. Thus only in the first case do the limit-points unfold smoothly to their limiting values.

In Fig. 19 the values of the secondary bifurcation parameters have been changed so that the steady-state diagram in the complete mixing case (the black line) contains one Hopf bifurcation point, called the 'I+1 $\mathrm{H}^{\prime}$ steady-state type in [18]. (Note that the periodic solution branch is shown). The incomplete mixing steady-state diagrams are all of

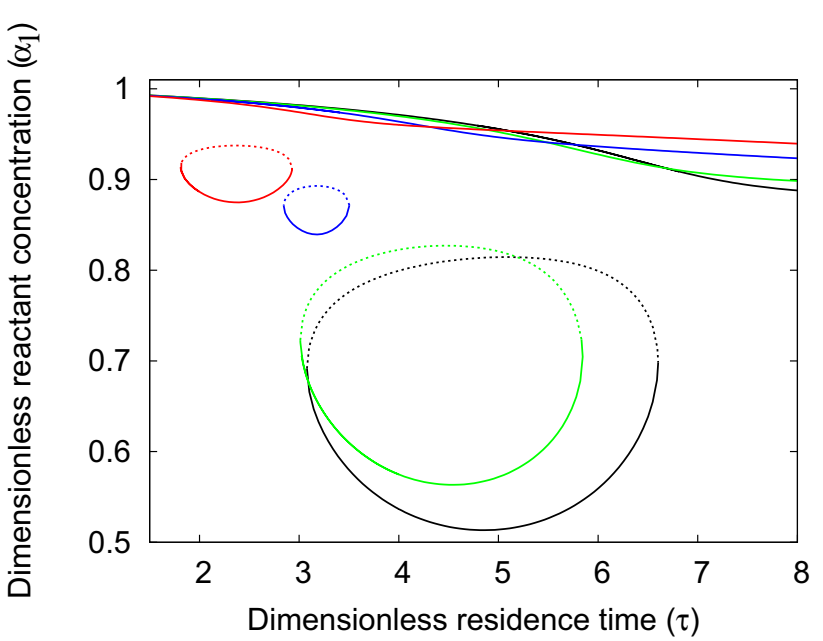

Fig. 17 Steady-state diagrams. In the well mixed case (black line) the steady-state diagram consists of an isola. The green $(\varepsilon=0.1)$, blue $(\varepsilon=0.2)$, and red $(\varepsilon=0.3)$ curves represent cases of incomplete mixing. Secondary bifurcation parameter values: $\left(\kappa_{2}, \beta_{0}\right)=(0.92,0.004)$. Other parameter values as in Fig. 1

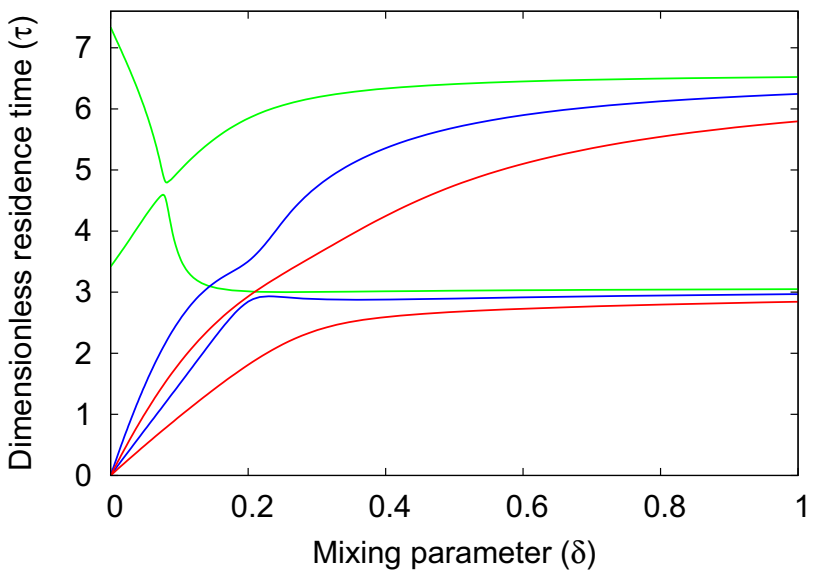

Fig. 18 Unfolding diagram for the limit points in Fig. 17

type of 'I', i.e. the presence of incomplete mixing has eliminated the Hopf bifurcation point.

In Fig. 20 the values of the secondary bifurcations parameters are identical those used in Fig. 19. However, the value of the mixing parameter has been increased from $\delta=0.2$ to $\delta=5$. Unlike in Fig. 19 the incomplete mixing steady-state diagrams are now of type ' $I+1 \mathrm{H}$ '. In this case mixing is sufficiently strong to overcome the negative effects of the stagnant regions. The location of the Hopf bifurcation point and the emanating branch of periodic solutions are more clearly seen in Fig. 20b.

Figure 20 shows that when $\delta=5$ the incomplete mixing steady-state diagrams have one Hopf bifurcation point, whereas Fig. 19 shows that when $\delta=0.2$ they contain no

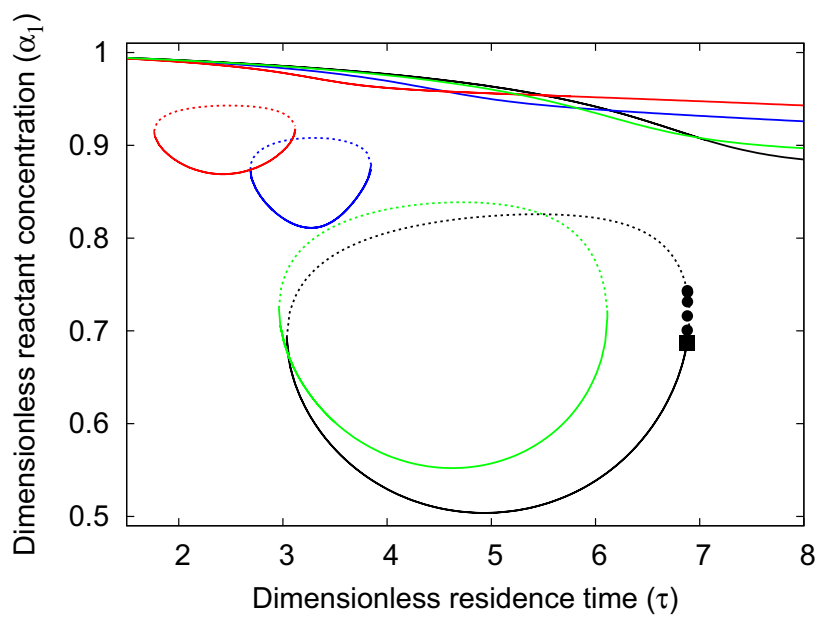

Fig. 19 Steady-state diagrams. In the well mixed case (black line) the steady-state diagram consists of an isola with one Hopf bifurcation. The green $(\varepsilon=0.1)$, blue $(\varepsilon=0.2)$, and red $(\varepsilon=0.3)$ curves represent cases of incomplete mixing. Secondary bifurcation parameter values: $\left(\kappa_{2}, \beta_{0}\right)=(0.912,0.0032)$. Other parameter values as in Fig. 1 


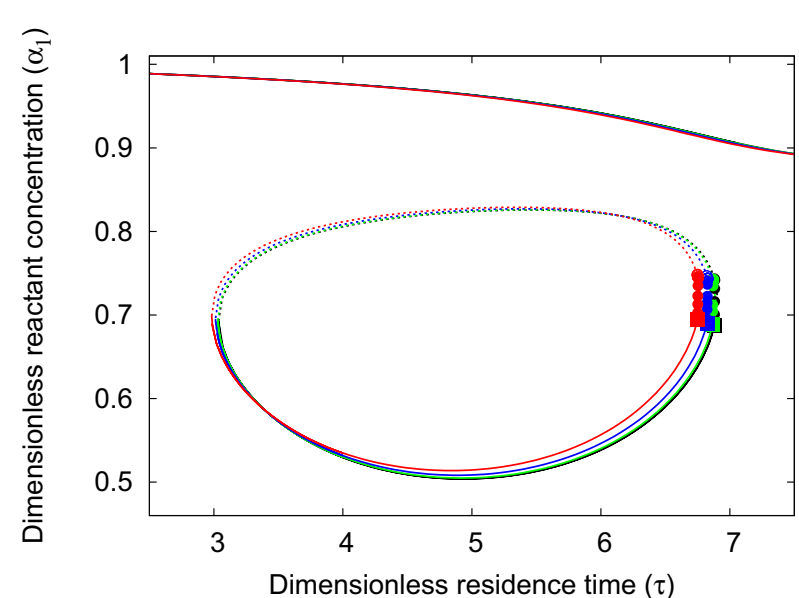

(a)

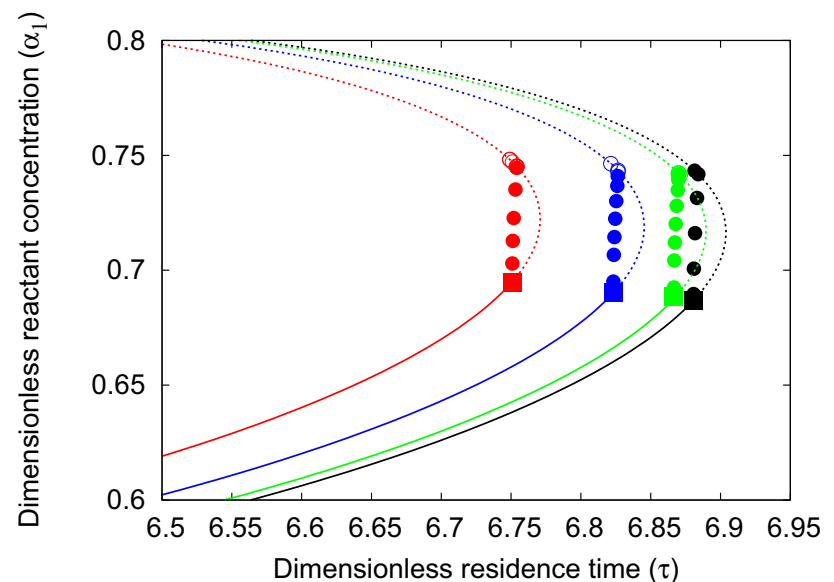

(b) Blow-up of (a)

Fig. 20 Steady-state diagrams. In the well mixed case (black line) the steady-state diagram consists of an isola with one Hopf bifurcation. The green $(\varepsilon=0.1)$, blue $(\varepsilon=0.2)$, and red $(\varepsilon=0.3)$ curves represent cases of incomplete mixing. Parameter values: $\left(\kappa_{2}, \beta_{0}\right)=(0.912,0.0032), \delta=5$. Other parameter values as in Fig. 1

Hopf bifurcation points. We deduce that as the value of the mixing parameter is reduced from $\delta=5$ that there must be a critical value at which the Hopf bifurcation point is removed from the steady-state diagram.

To investigate this in Fig. 21 we unfold the Hopf bifurcation points from Fig. 20 with the mixing parameter $(\delta)$. For each size of the stagnant region $(\varepsilon)$ there is a critical value of the mixing parameter below which the Hopf bifurcation points are eliminated. These critical values are: $\delta_{\mathrm{cr}}=0.477(\varepsilon=0.1) ; \delta_{\mathrm{cr}}=1.205(\varepsilon=0.2)$; and $\delta_{\mathrm{cr}}=1.993(\varepsilon=0.3)$. The critical values increase as the size of the stagnant region $(\varepsilon)$ increases.

Magnifying the Hopf unfolding locus in the vicinity of the critical value of the mixing parameter reveals that the unfolding is is more complicated than suggested by Fig. 21. In Fig. 22 it can be seen that there are two degenerate Hopf bifurcation points. The circle indicates a

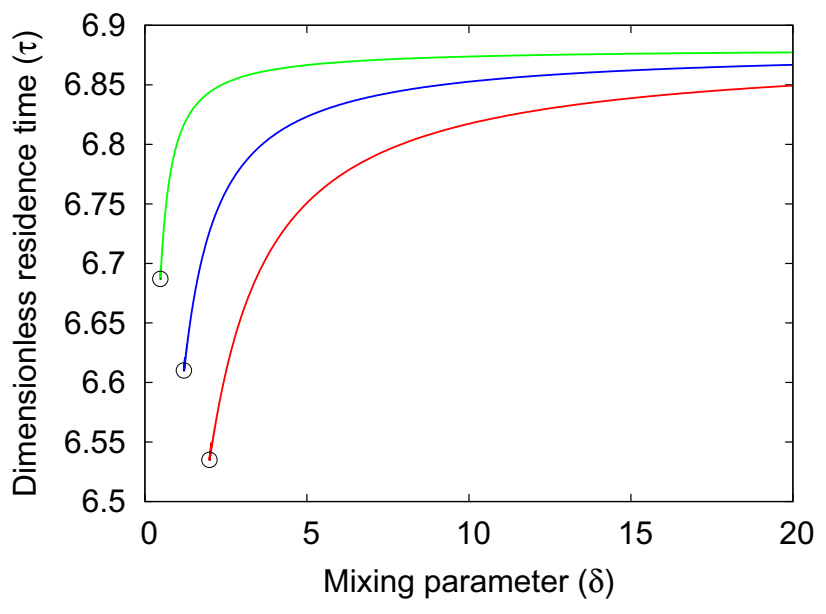

Fig. 21 Unfolding diagram for the Hopf bifurcation points in Fig. 20

double-Hopf bifurcation, this is the degenerate bifurcation indicated on Fig. 21. The diamond indicates a double-zero eigenvalue bifurcation. One of the Hopf bifurcation points generated by the double-Hopf bifurcation is terminated at the double-zero eigenvalue bifurcation. Thus there is a range of values for the mixing parameter over which incomplete mixing increases the number of Hopf bifurcations to two. This range is given by: $0.477<\delta<0.491$ $(\varepsilon=0.1) ; 1.205<\delta<1.237(\varepsilon=0.2)$; and1.993 $<\delta<2.056$ $(\varepsilon=0.3)$. We note that the value of the mixing parameter used in Fig. 20 ( $\delta=5$ ) is larger than any of the values at the double-zero eigenvalue bifurcation, which is why only one Hopf bifurcation point is observed on Fig. 20.

Bifurcation theory ensures that there is a locus of homoclinic bifurcations emerging from the double zero eigenvalue point [15]. We have not investigated the homoclinic bifurcation points as they are not the focus of this study.

To conclude, in this section we have seen that as incomplete mixing is introduced into the model, i.e. reducing the mixing parameter from $\delta=\infty$, that an additional Hopf bifurcation is eventually created at a double-zero eigenvalue bifurcation (Fig. 22). As the mixing parameter is reduced further both Hopf bifurcation points are destroyed at a double-zero eigenvalue bifurcation (Fig. 22).

\subsubsection{Mushroom steady-state diagram}

In this section we set the values of the secondary bifurcation parameters, the scaled decay parameter $\kappa_{2}$ and the scaled catalyst concentration in the feed $\beta_{0}$, so that the steady-state diagram in the case of perfect mixing is the mushroom steady-state diagram, called type ' $M$ ' in [18]. In Fig. 23 the perfect mixing steady-state diagram is indicated by the the black line. Its distinguishing feature is that it consists of a single solution branch containing 


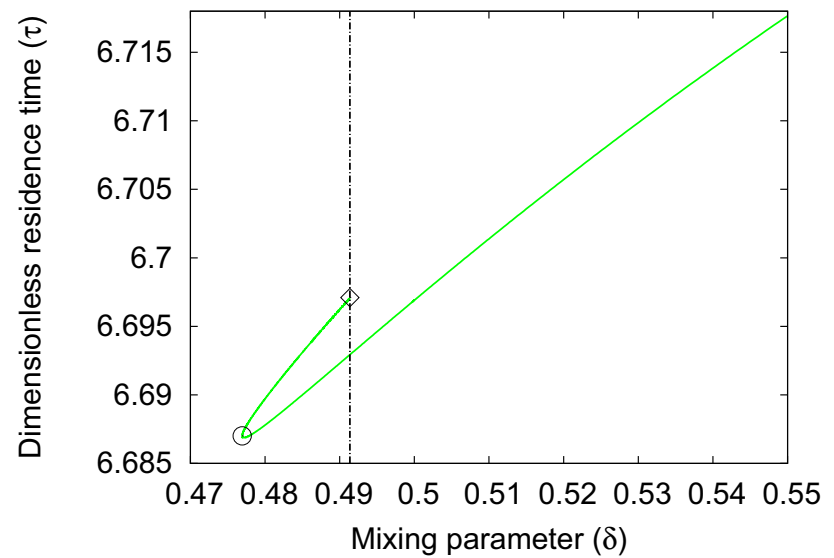

(a) Blow-up of figure $21(\varepsilon=0.1)$.

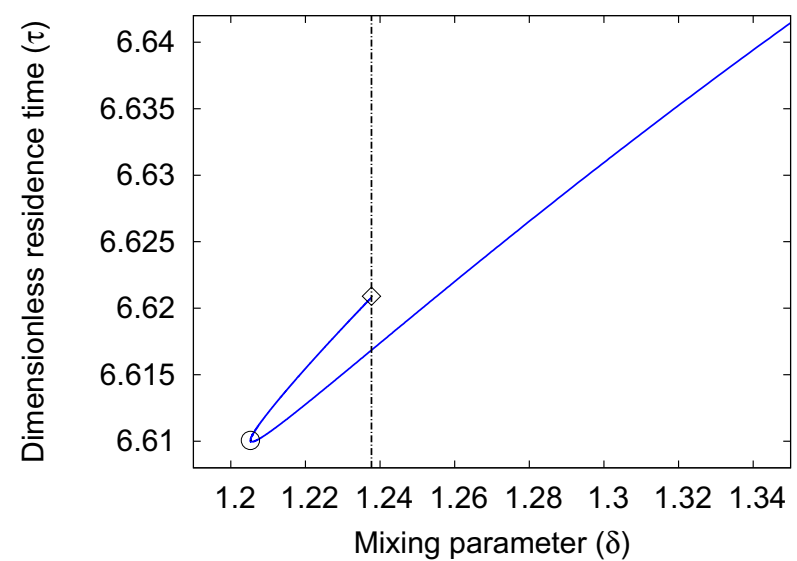

(b) Blow-up of figure $21(\varepsilon=0.2)$.

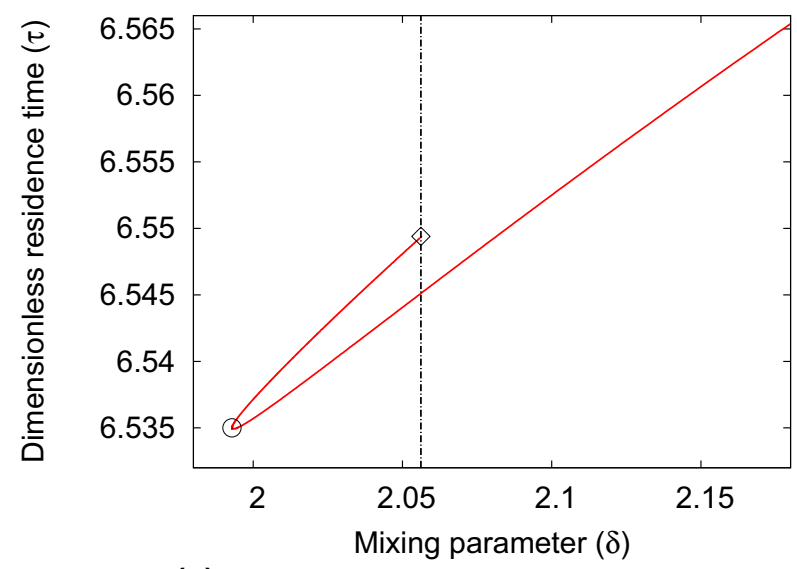

(c) Blow-up of figure $21(\varepsilon=0.3)$.

Fig. 22 Blow-up of the unfolding diagram for the Hopf bifurcation points in Fig. 21. The circle and diamond points denote double-Hopf and double-zero bifurcation points respectively. The double Hopf bifurcations are at: $\left(\delta_{\mathrm{cr}}, \tau_{\mathrm{cr}}\right)=(0.477,6.687)$, $\left(\delta_{\mathrm{cr}}, \tau_{\mathrm{cr}}\right)=(1.205,6.610)$ and $\left(\delta_{\mathrm{cr}}, \tau_{\mathrm{cr}}\right)=(1.993,6.535)$. The doublezero eigenvalue bifurcations are at: $\left(\delta_{\mathrm{cr}, 2}, \tau_{\mathrm{cr}, 2}\right)=(0.491,6.697)$, $\left(\delta_{\mathrm{cr}, 2}, \tau_{\mathrm{cr}, 2}\right)=(1.237,6.620)$ and $\left(\delta_{\mathrm{cr}, 2}, \tau_{\mathrm{cr}, 2}\right)=(2.056,6.549)$. In each case the size of the stagnant regions are: $\varepsilon=0.1, \varepsilon=0.2$ and $\varepsilon=0.3$ respectively

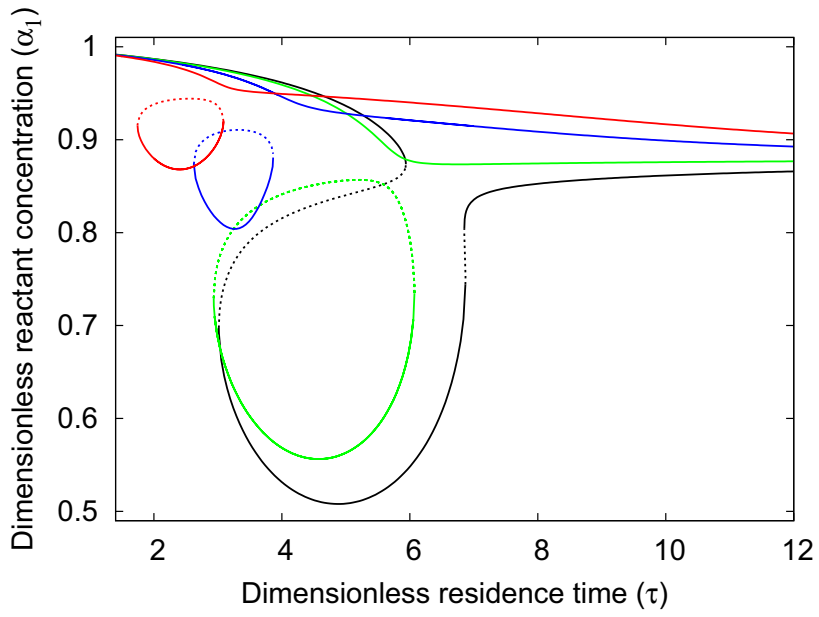

Fig. 23 Steady-state diagrams. In the well mixed case (black line) the steady-state diagram consists of a mushroom. The green ( $\varepsilon=0.1)$, blue $(\varepsilon=0.2)$, and red $(\varepsilon=0.3)$ curves represent cases of incomplete mixing. Secondary bifurcation parameter values: $\left(\kappa_{2}, \beta_{0}\right)=(0.92,0.005)$. Other parameter values as in Fig. 1

four limit-point bifurcations. These are arranged in such a way that the maximum number of steady-state solutions is three.

In Fig. 23 none of the incomplete mixing steady-state diagrams are of the mushroom type; they are all of the isola type. The effect of incomplete mixing has been to remove two limit points from the steady-state diagram, in the process pinching off the 'mushroom' and forming an isola.

In Fig. 24 the value of the mixing parameter is increased from $\delta=0.2$ to $\delta=1$. Now the steady-state diagrams for

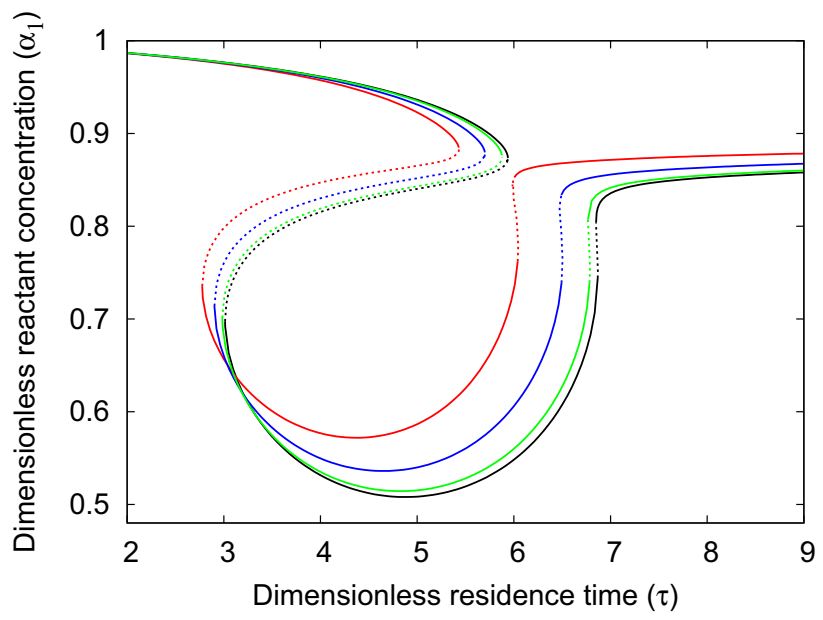

Fig. 24 Steady-state diagrams. In the well mixed case (black line) the steady-state diagram consists of a mushroom. The green $(\varepsilon=0.1)$, blue $(\varepsilon=0.2)$, and red $(\varepsilon=0.3)$ curves represent cases of incomplete mixing. Parameter value: $\delta=1$. Other parameter values as in Fig. 23 
all the imperfect mixing models are of type ' $M$ '. Thus when $\delta=1$ the incomplete mixing steady-state diagrams have four limit points whereas when $\delta=0.2$ they have two limit points.

To investigate the transition in the steady-state diagrams as the value of the mixing parameter is changed, Fig. 25 unfolds the limit points exhibited

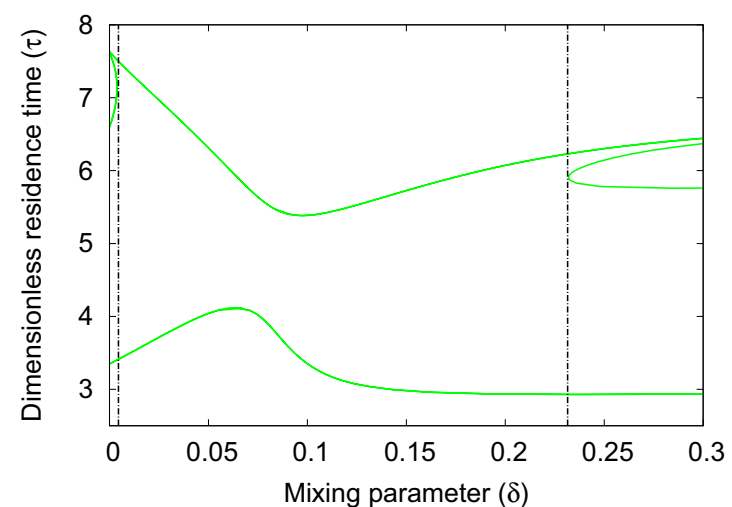

(a) The case $\varepsilon=0.1$.

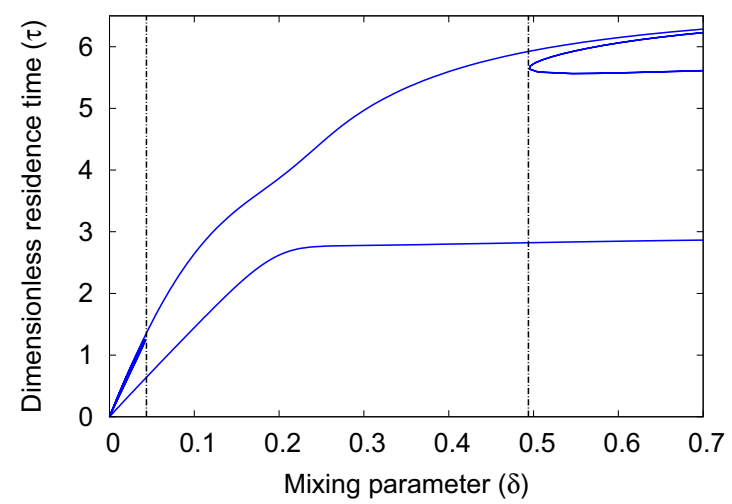

(c) The case $\varepsilon=0.2$.

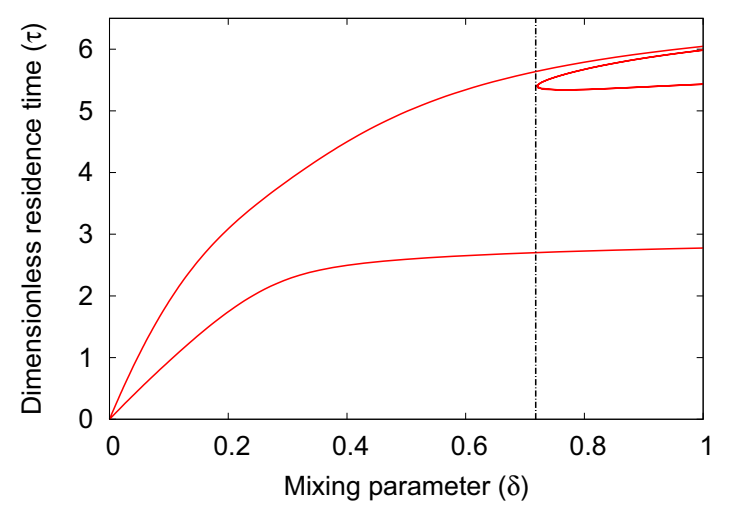

(e) The case $\varepsilon=0.3$

Fig. 25 Unfolding diagram for the limit points in Fig. 24. In figures $\mathbf{a}$ and $\mathbf{b}, \varepsilon=0.1$, the critical values are: $\left(\delta_{\mathrm{cr}, 1}, \tau_{\mathrm{cr}, 1}\right)=(0.232,5.927)$, and $\left(\delta_{\mathrm{cr}, 2}, \tau_{\mathrm{cr}, 2}\right)=(0.0037,7.179)$. In figures $\mathbf{c}$ and $\mathbf{d}, \varepsilon=0.2$, by the incomplete mixing steady-state diagrams in Fig. 24. (Note that to improve the clarity of the figures the unfoldings are not all shown starting at $\delta=1$.) This figure shows that, in all three cases, as the mixing parameter is decreased from $\delta=1$ there is a bifurcation point, $(\delta, \tau)=\left(\delta_{\mathrm{cr}, 1}, \tau_{\mathrm{cr}, 1}\right)$, at which two limit points are eliminated from the steady-state diagram

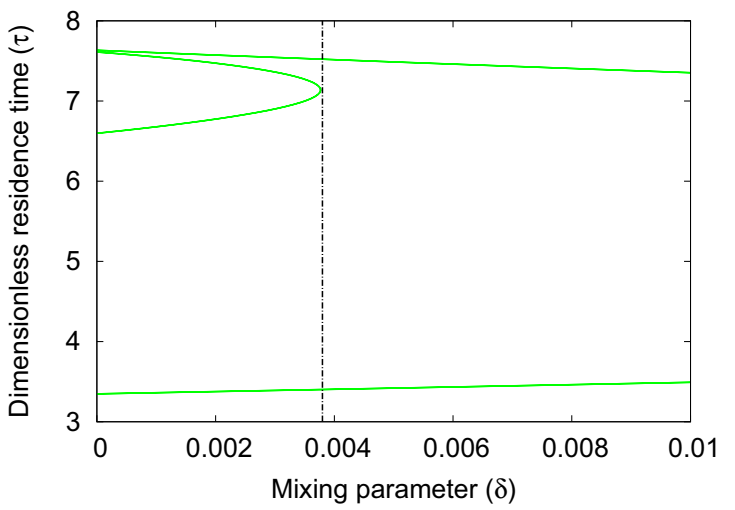

(b) Blow-up of (a).

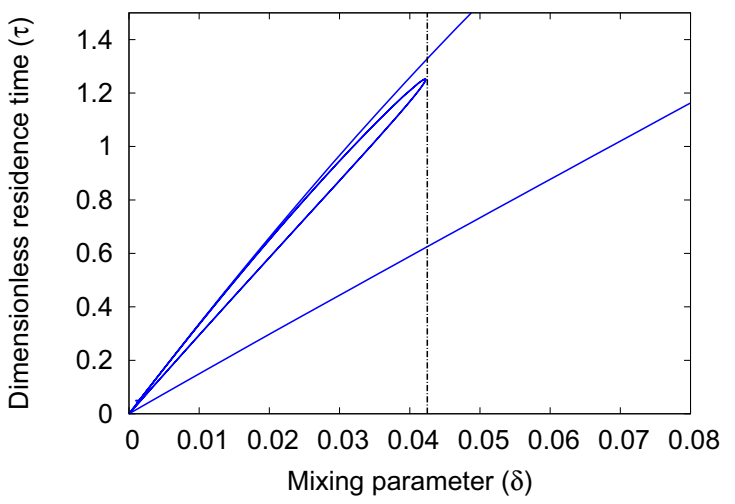

(d) Blow-up of (c).

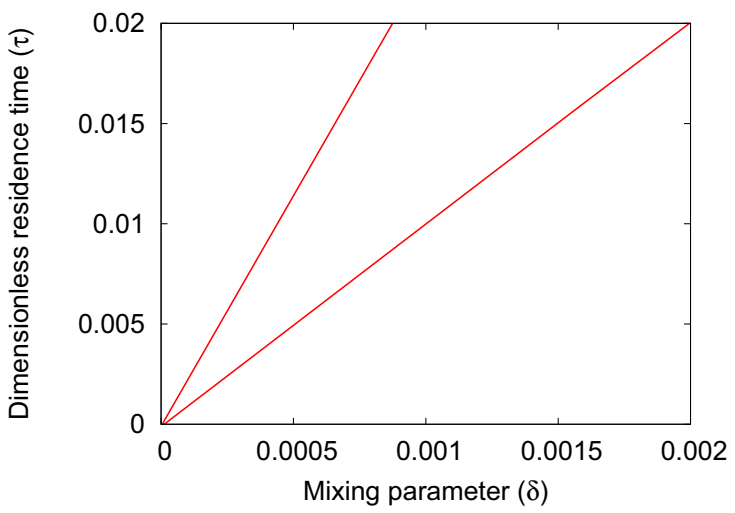

(f) Blow-up of (e).

the critical values are: $\left(\delta_{\mathrm{cr}, 1}, \tau_{\mathrm{cr}, 1}\right)=(0.495,5.638)$, and. $\left(\delta_{\mathrm{cr}, 2}, \tau_{\mathrm{cr}, 2}\right)=(0.042,1.251)$. In figures $\mathbf{e}$ and $\mathbf{f}, \varepsilon=0.3$, the critical value is: $\left(\delta_{\mathrm{cr}}, \tau_{\mathrm{cr}}\right)=(0.719,5.400)$ 
due to an isola singularity. For the two steady-state diagrams with the smaller sizes of the stagnant region, i.e. $\varepsilon=0.1$ and $\varepsilon=0.2$, there is a second isola singularity, $(\delta, \tau)=\left(\delta_{\mathrm{cr}, 2}, \tau_{\mathrm{cr}, 2}\right)$, at which two limit points are created. These figures explain why the structure of the steady-state diagram in Fig. 23 is a mushroom for the well-stirred model but an isola for the imperfect mixing models.

To conclude, in this section we have seen that incomplete mixing can both create and destroy limit points.

\subsubsection{Isola with breaking wave steady-state diagram}

In this section we set the values of the secondary bifurcation parameters, the scaled decay parameter $\kappa_{2}$ and the scaled catalyst concentration in the feed $\beta_{0}$, so that the steady-state diagram in the case of perfect mixing is the isola with breaking wave steady-state diagram with two Hopf bifurcation points, called type 'I+B+2H' in [18]. In Fig. 26 the perfect mixing steady-state diagram is indicated by the the black line. Its distinguishing feature is that it consists of two disconnected solution branches: an isola and a breaking wave.

In Fig. 26 the steady-state diagrams for the three incomplete mixing models are all of the type of ' $I+1 \mathrm{H}$ ', i.e. the consequences of poor mixing is the removal of two limit points, along the breaking-wave branch, and one Hopf bifurcation point.

In Fig. 27 the value of the mixing parameter is increased from $\delta=0.2$ to $\delta=2$. Now all of the incomplete

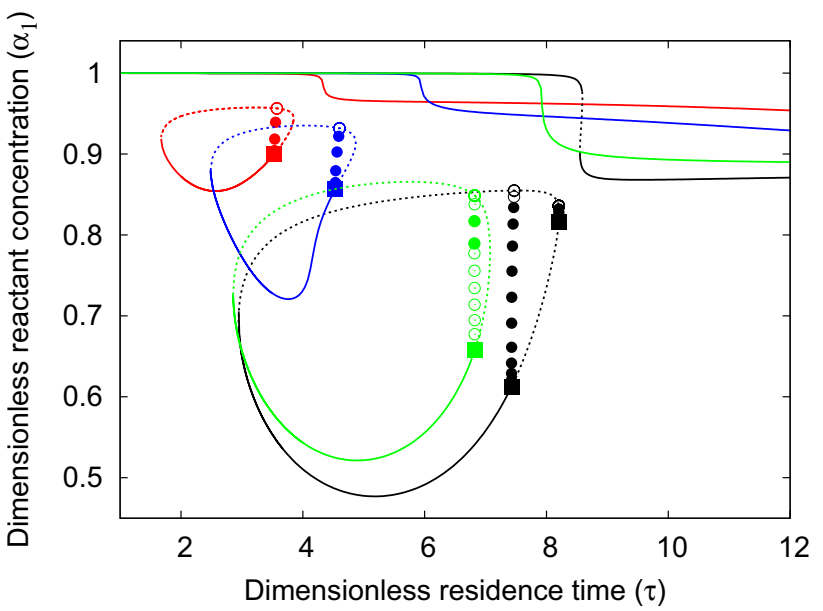

Fig. 26 Steady-state diagrams. In the well mixed case (black line) the static steady-state diagram consists of the isola with breaking wave type. The green $(\varepsilon=0.1)$, blue $(\varepsilon=0.2)$, and red $(\varepsilon=0.3)$ curves represent cases of incomplete mixing. Secondary bifurcation parameter values: $\left(\kappa_{2}, \beta_{0}\right)=(0.885,0.000025)$. Other parameter values as in Fig. 1

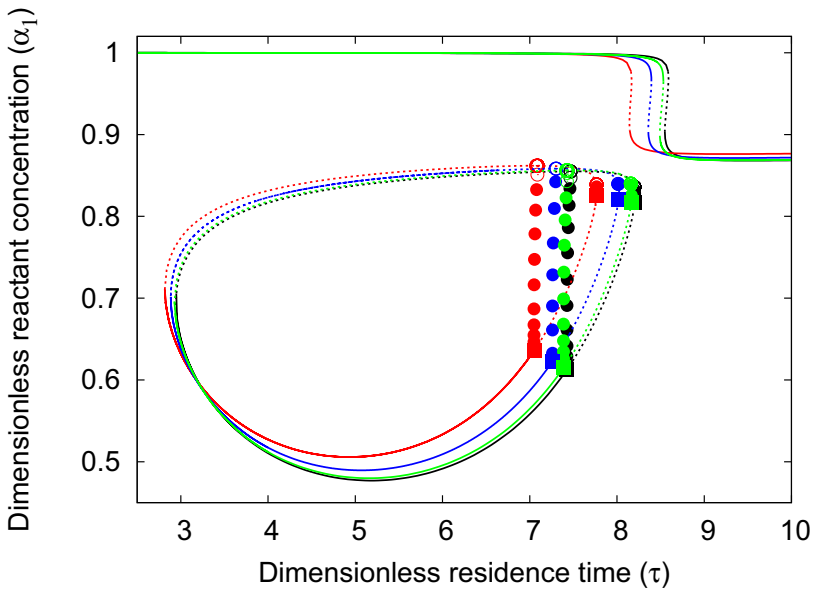

(a)

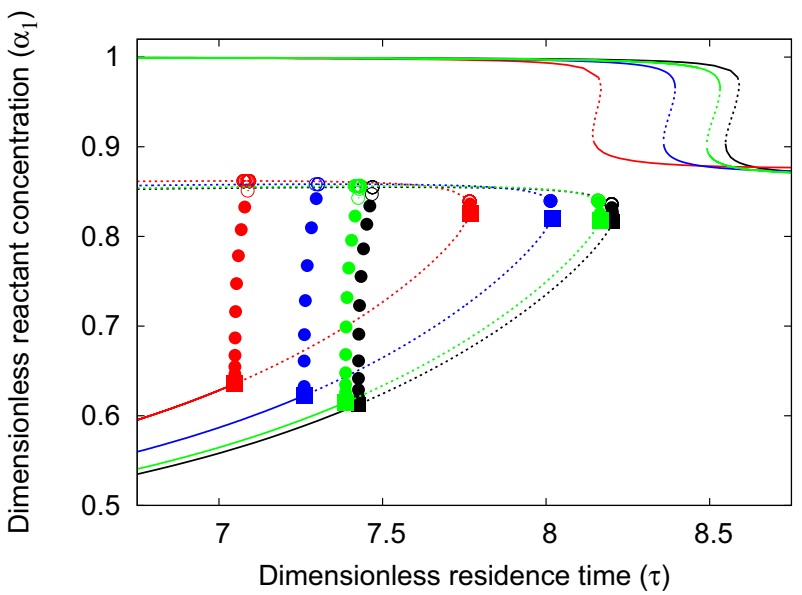

(b) Blow-up of (a).

Fig. 27 Steady-state diagrams. In the well mixed case (black line) the static steady-state diagram consists of the isola with breaking wave type. The green $(\varepsilon=0.1)$, blue $(\varepsilon=0.2)$, and red $(\varepsilon=0.3)$ curves represent cases of incomplete mixing. Parameter value: $\delta=2$ (for incomplete mixing models). Other parameter values as in Fig. 26

mixing steady-state diagrams are of type the 'I+B+2 $\mathrm{H}$ '. Thus when $\delta=2$ the incomplete mixing steady-state diagrams have four limit points and two Hopf bifurcation points whereas when $\delta=0.2$ they have two limit points and one Hopf bifurcation point.

From Figs. 26 and 27 we deduce that as the mixing parameter is reduced there must a critical value at which a Hopf bifurcation is destroyed and a second critical value at which the two limit points on the breaking wave solution branch are destroyed. To investigate this, Fig. 28 shows unfoldings of the bifurcation points on Fig. 27.

Figure 28a shows that the limit-points on the isola unfold smoothly as the mixing parameter is decreased. However, Fig. 28b shows that the two limit points on the breaking wave are destroyed by a cusp singularity at a critical value of the mixing parameter $\left(\delta_{\mathrm{cr}}\right)$. Thus for $\delta<\delta_{\mathrm{cr}}$ 
Table 1 Critical values of limit (circle) and Hopf bifurcation (diamond) points shown in Fig. 28

\begin{tabular}{llll}
\hline$\varepsilon$ & 0.1 & 0.2 & 0.3 \\
\hline$\left(\delta_{\mathrm{cr}}, \tau_{\mathrm{cr}}\right)$ & $(0.241,8.079)$ & $(0.493,7.713)$ & $(0.692,7.314)$ \\
$\left(\delta_{\mathrm{DZE}, 1}, \tau_{\mathrm{DZE}, 1}\right)$ & $(0.146,6.672)$ & $(0.852,7.641)$ & $(1.28,7.455)$ \\
$\left(\delta_{\mathrm{DZE}, 2}, \tau_{\mathrm{DZE}, 2}\right)$ & $(0.415,7.868)$ & - & - \\
\hline
\end{tabular}

there is no breaking wave. The values for $\delta_{\mathrm{cr}}$ are provided in Table 1.

Figure $28 \mathrm{c}$ shows that for the cases $\varepsilon=0.2$ and $\varepsilon=0.3$, as the mixing parameter is reduced, there is a single double zero eigenvalue bifurcation $\left(\delta_{\mathrm{DZE}}\right)$ that eliminates one Hopf bifurcation. For values of the mixing parameter lower than this, i.e. $\delta<\delta_{\mathrm{DZE}}$, there is only one Hopf bifurcation point. When $\varepsilon=0.1$ there are two double-zero eigenvalue bifurcation, with $\delta_{\mathrm{DZE}, 1}>\delta_{\mathrm{DZE}, 2}$. If the mixing parameter is sufficiently small, i.e. $\delta<\delta_{\mathrm{DZE}, 2}$, then are no Hopf bifurcation points on the isola for the case $\varepsilon=0.1$. As was the case in Sect. 3.3.3 we have not investigated the locus of homoclinic bifurcations emerging from the double zero eigenvalue point as they are not the focus of this study.

The values for $\delta_{\mathrm{DZE}, \text { a }}$ are provided in Table 1 .

To conclude. As incomplete mixing is introduced into the model, i.e. reducing the mixing parameter from $\delta=\infty$, an additional Hopf bifurcation is eventually created at a double-zero eigenvalue bifurcation. As the mixing parameter is reduced further-depending upon the size of the stagnant region $(\varepsilon)$ - then either one or both of these Hopf bifurcations are removed (Fig. 28b).

The effect of introducing incomplete mixing is to remove two limits points on the breaking wave-changing it into a unique steady-state diagram.

\section{Conclusions}

In this paper we have extended our earlier investigation of the reaction schemes 1 and 2 [18] to incorporate the effects of partial segregation inside the reactor due to imperfect mixing.

The extended model has four secondary bifurcation parameters and one tertiary bifurcation parameter. Two of the secondary bifurcation parameters are associated with the well-mixed model, $\kappa_{2}$ and $\beta_{0}$. Two of the secondary parameters are associated with the mixing model, $\varepsilon$ and $\delta$ ). Unlike our earlier study it is no longer possible to find a comprehensive analysis across the entirety of the parameter space.

Instead we have chosen to investigate how poor mixing can change the steady-state diagram of the system. To do this we selected the values for the secondary

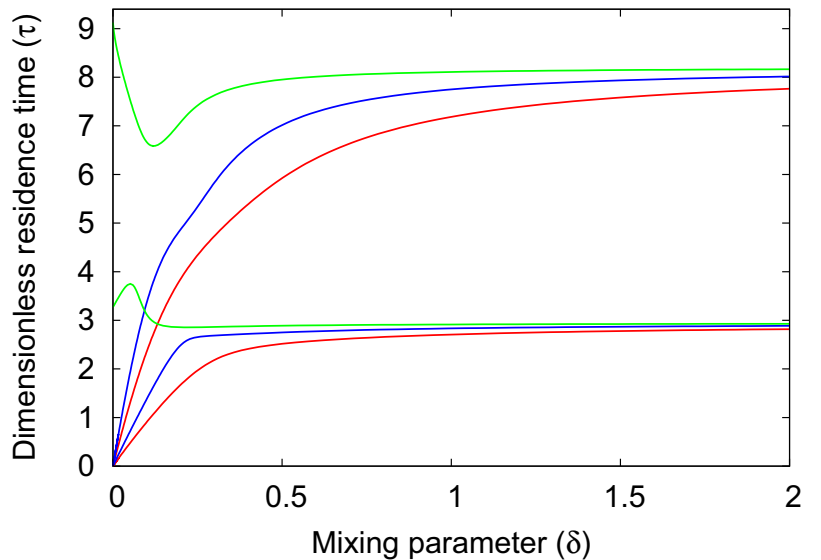

(a) Unfolding the isola limit points.

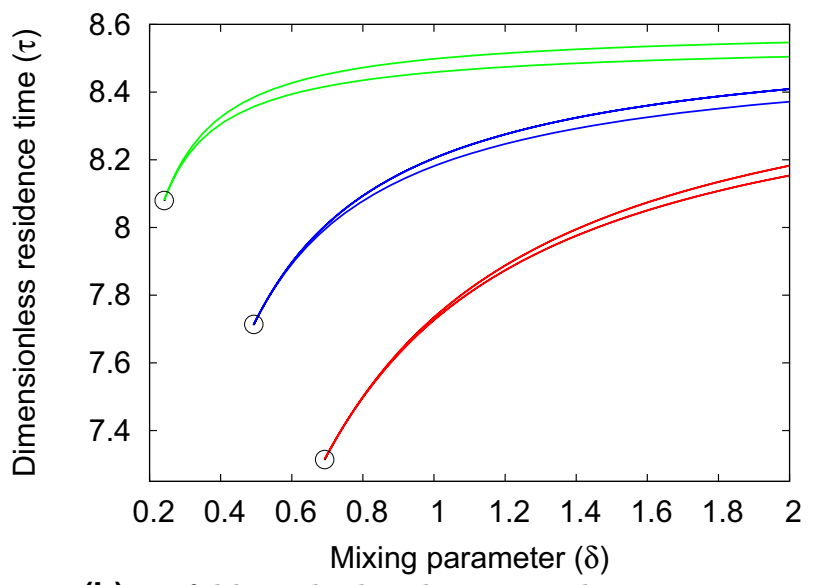

(b) Unfolding the breaking wave limit points.

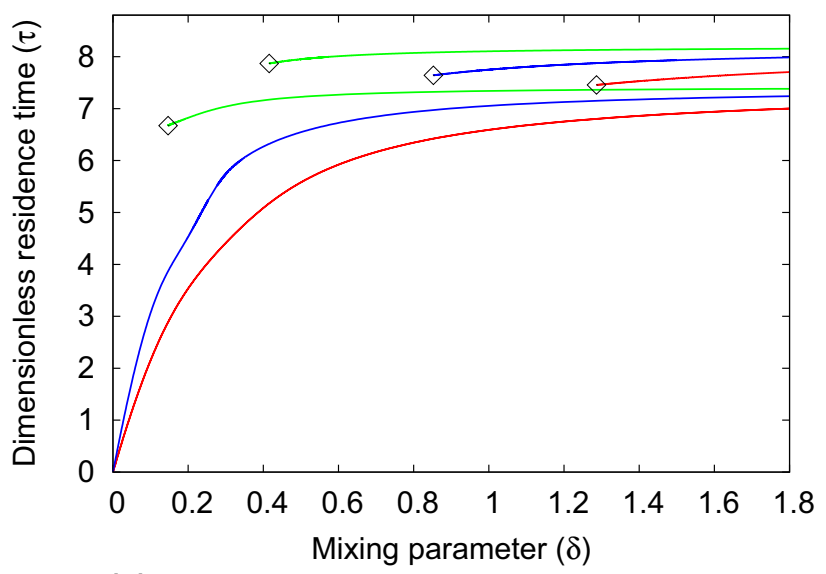

(c) Unfolding the Hopf bifurcation points.

Fig. 28 Unfolding diagram for the bifurcation points in Fig. 26. The green $(\varepsilon=0.1)$, blue $(\varepsilon=0.2)$, and red $(\varepsilon=0.3)$ curves represent cases of incomplete mixing. The location of the critical values is given in Table 1

bifurcation parameters $\kappa_{2}$ and $\beta_{0}$ so as to pick one of the five generic static steady-state diagrams that are exhibited by the weakly mixed model. We then investigated how 
the response diagram changes for small values of the mixing parameter $(\delta)$ for three sizes of the stagnant region $(\varepsilon=0.1, \varepsilon=0.2$ and $\varepsilon=0.3)$. We showed that poor mixing can lead to the destruction of both Hopf bifurcation points and limit points. In some circumstances poor mixing can create additional Hopf bifurcation points.

For instance, in Sect. 3.3.2 the steady-state diagram for the well mixed model is a breaking wave with an isola whereas for systems subject to poor mixing it was an isola. Furthermore, in Sect. 3.3.5 the steady-state diagram for the well mixed model is a breaking wave with an isola containing two Hopf bifurcation points whereas for systems subject to poor mixing it was an isola containing a single Hopf bifurcation point.

Although formally the perfect mixing model is obtained only in the limit that $\delta \rightarrow \infty$ we have shown in practice incomplete mixing does not occur once the mixing parameter crosses some finite threshold. When the mixing parameter is below this threshold, i.e. it is sufficiently small, we have shown that imperfect mixing has the ability to modify the static and dynamic bifurcation behaviour exhibited in the model.

Acknowledgements During this work Ahmed Msmali was a PhD student at the University of Wollongong. He gratefully acknowledges the award of a PhD scholarship by the Saudi government and the University of Om Al-qura (Saudi Arabia).

\section{Compliance with ethical standards}

Conflict of interest On behalf of all authors, the corresponding author states that there is no conflict of interest.

\section{References}

1. Ajbar A, Alhumaizi K (2012) Dynamics of the chemostat, 1 st edn. CRC Press, Boca Raton

2. Brindley J, Kaas-Petersen C, Merkin JH, Scott SK (1989) A simple model for sustained oscillations in isothermal branched-chain or autocatalytic reactions in a well-stirred open system III. Multiple stationary states and Hopf bifurcations. Proc R Soc Lond 417:463-496

3. Corrigan TE, Beavers WO (1968) Dead space interaction in continuous stirred tank reactors. Chem Eng Sci 23:1003-1006

4. Epstein IR, Pojman JA (1998) An introduction to nonlinear chemical dynamics, 1st edn. Oxford University Press, Oxford
5. Ermentrout B (2002) Simulating, analyzing, and animating dynamical systems. Society for Industrial and Applied Mathematics, Philadelphia

6. Fogler HS (1999) Elements of chemical reaction engineering, 3rd edn. Prentice Hall, Upper Saddle River

7. Ganapathisubramanian N (1991) Tristability in the iodate-As(iii) chemical system arising from a model of stirring and mixing effects. J Chem Phys 95:3005-3008

8. Ganapathisubramanian N (1992) Tristability and related nonlinear dynamic phenomena in the iodate-Arsenic(iii) chemical in a CSTR with a satellite reactor: a computational study. J Phys Chem 96:4446-4450

9. Gray BF, Roberts MJ (1988) A method for the complete qualitative analysis of two coupled ordinary differential equations dependent on three parameters. Proc R Soc Lond 416:361-389

10. Gray P, Scott SK (1990) Chemical oscillations and instabilities: non-linear chemical kinetics, 1st edn. Oxford University Press, Oxford

11. Györgyi L, Field RJ (1989) Aperiodicity resulting from two-cycle coupling in the Belousov-Zhabotinskii reaction. III. Analysis of a model of the effect of spatial inhomogeneities at the input ports of a continuous-flow, stirred tank reactor. J Chem Phys 91:6131-6141

12. Hsu TJ, Lee DJ (1995) Macromixing effects on the KumpinskyEpstein model of a chlorite-iodide reaction in a stirred tank. J Chem Phys 102:8274-8276

13. Hsu TJ, Mou CY, Lee DJ (1996) Macromixing effects on the grayscott model in a stirred reactor. Chem Eng Sci 51:2589-2594

14. Kumpinsky E, Epstein IR (1985) A model for stirring effects on transitions in bistable chemical systems. J Chem Phys 82:53-57

15. Kuznetsov YA (1995) Elements of applied bifurcation theory, 1st edn. Springer, New York

16. Merkin JH, Needham DJ, Scott SK (1985) A simple model for sustained oscillations in isothermal branched-chain or autocatalytic reactions in a well stirred open system. I. Stationary states and local stabilities. Proc R Soc Lond 398:81-100

17. Merkin JH, Needham DJ, Scott SK (1985) A simple model for sustained oscillations in isothermal branched-chain or autocataIytic reactions in a well stirred open system. II. Limit cycles and non-stationary states. Proc R Soc Lond 398:101-116

18. Msmali AH, Nelson Ml, Edwards MP (2014) Quadratic autocatalysis with non-linear decay. J Math Chem 52:2234-2258

19. Shuler ML, Kargi $F$ (2002) Bioprocess engineering, 2nd edn. Prentice Hall international series in the physical and chemical engineering sciences. Prentice Hall, New Jersey

20. Strogatz Steven H (2000) Nonlinear dynamics and chaos: with applications to physics, biology, chemistry and engineering. Westview Press, Boulder

Publisher's Note Springer Nature remains neutral with regard to jurisdictional claims in published maps and institutional affiliations. 